\title{
Optical knots and contact geometry I. From Arnol'd inequality to Ranada's dyons
}

\author{
Arkady L. Kholodenko \\ 375 H.L.Hunter Laboratories, Clemson University, Clemson, SC 29634-0973,USA
}

\begin{abstract}
Recently there had been a great deal of activity associated with various schemes of designing both analytical and experimental methods describing knotted structures in electrodynamics and in hydrodynamics. The majority of works in electrodynamics were inspired by the influential paper by Ranada (1989) and its subsequent refinements. In this work and in its companion we reanalyze Ranada's results using methods of contact geometry and topology. Not only our analysis allows us to reproduce his major results but, in addition, it provides opportunities for considerably extending the catalog of known knot types. Furthermore, it allows to reinterpret both the electric and magnetic charges purely topologically thus opening the possibility of treatment of masses and charges in Yang-Mills and gravity theories also topologically. According to (now proven) Thurston's geometrization conjecture complements of all knots/links in $S^{3}$ are spaces of positive, zero or negative curvature. This means that spaces around our topological masses/charges are curved. This fact is essential for design of purely topological theories of gravity, electromagnetism and strong/weak interactions.

PACS numbers : 11.15 Yc; 11.27.+d; 11.30.-j; 42, 45.20 Jj; 47
\end{abstract}

\section{Introduction}

\subsection{Motivation and background}

In 1858 Herman von Helmholtz published a paper (Helmholtz 1858) in which he demonstrated that ideal incompressible constant density fluid should contain vortex excitations which are stable1. This result had attracted attention of Maxwell, Tait and Thomson (Lord Kelvin). In particular, Thomson developed an atomic vortex theory in which the rigidity of Hemholtz vortices was related to supposed indivisibility of atoms. Different atoms are expected to have different vortex types. To classify different vortex types Tait developed what is now known as knot theory while Maxwell developed some applications of these results by Thomson and Tait to chemical reactions. Subsequently, in 1883, J.J.Thomson (the man who discovered the electron) wrote a monograph entitled "A Treatise on the Motion of Vortex Rings" (Thompson 1883) in which he further developed the theory by extending

\footnotetext{
${ }^{1}$ In fact, they were believed to be stable/permanent and indivisible (Hemholtz 1858, Thompson 1868)
} 
it to the linked vortices and streamlined Maxwell's results. Subsequent studies done century later demonstrated the possibility of existence of torus knots and links in ideal fluids (Ricca 2001). Although elementary vortices in fluids were experimentally known for quite some time, the existence of knotted structures, although allowed theoretically, escaped experimental verification till 2013 (Kleckner and Irvine 2013). To prove the existence of Helmholtz vortex structures Tait designed an apparatus capable of producing smoke rings. The design of apparatus by Tait was so successful that he was able to demonstrate that (Lomonaco 1996):

1.The vortex rings can exist for a long time.

2.On collision the rings were capable of scattering from each other as if they were made of rubber.

3.The rings exhibited some vibration modes around their circular form.

4.On each attempt to cut the smoke rings with a knife, the smoke rings would wriggle around the knife without breaking.

Such a situation with vortices remained basically unchanged till 2013. It should be noted that in 2013 the results were obtained in the lab (not detected yet in Nature) while the theory allows the existence of knotted/linked structures in Nature. Based on the results just presented, it is clear that existence of knotted/linked structures depends upon:

a) the way these structures are prepared;

b) the way these structures are detected;

c) the stability of these structures from the moment they were created to the moment they are detected.

Evidently, c) is mainly theoretical issue while a) and b) are experimental. In this paper by extending some latest results from hydrodynamics of incompressible ideal fluids to electrodynamics, we describe new classes of knotted/linked structures not present in the works by Ranada (and associates) and others e.g. (Kedia et al 2013). Although these new classes of knots/links have their origins in contact geometry/topology (Kholodenko 2013), they should not be confused with the optical knots discussed by Arnol'd (1986). Surely, mathematically they can be brought into correspondence with each other.

The development of correspondences between different subdisciplines of physics took place slowly and at different times. Perhaps the oldest, is the correspondence between the dynamics of ideal fluids and mechanics. A bit later the correspondence between geometrical optics and mechanics was established (Arnol'd 1989)2. Mechanics -hydrodynamics correspondence was exploited in great detail in the book by Arnol'd (Arnol'd and Khesin 1998). Surprisingly, it develops the formalism without much use of methods of contact geometry. This is surprising since Arnol'd was the major proponent of contact geometry. In yet another book by Arnol'd (Arnol'd 1984), in Chr.14, he stated that "Contact geometry is playing in optics and theory of wave propagation the same role as symplectic geometry for mechanics". This remark by Arnol'd (apparently) was left unnoticed in physics literature. In this paper and its companion we are making an attempt at eliminating the existing deficiency using

\footnotetext{
${ }^{2}$ Incidentally, Arnol'd optical knots have their origin in geometrical optics.
} 
general principles of contact geometry and topology outlined in our book (Kholodenko 2013). By doing so new directions in detecting of these new knotted structures are being suggested.

\subsection{Dynamically generated knotted and linked structures and Chern-Simons} topological field theory

Recent studies of dynamical systems revealed many instances in which knotted and linked structures had been dynamically generated (Birman and Williams 1983, Ghrist, Holmes and Sullivan 1997, Ghys 2007), detected and classified. Already mentioned correspondence between mechanics and fluid mechanics, mechanics and electromagnetism, mechanics and geometrical optics provides needed assurance for existence of knotted and linked structures, say, in elctromagnetism. This correspondence is unusual from the standpoint of currently existing opinion in physics literature. Indeed, beginning from the work by Witten (1989) it is widely accepted that only the non-Abelian version of the Chern-Simons (C-S) functional should be used for description of nontrivial knots and links. The word "nontrivial" suggests knots/links other than unknots, Hopf links and torus-type knots. The C-S functional emerges naturally from the theory of pure Yang-Mills (Y-M) fields. In fact, thanks to work of Floer (Donaldson 2002 ) it is possible to replace the nonperturbative treatment of 4-dimensional Y-M gauge theory by the analogous treatment of $3+1$ gauge theory in which the 4 dimensional Y-M functional is being replaced by the 3-dimensional C-S functional. This is in contrast with some papers in physics literature in which both the Y-M and C-S functionals are present in the initial action functional. Thus, because of this replacement, when treated nonperturbatively, theory of pure Y-M fields becomes topological. The C-S functional was already used for description of knotted dynamical flows by Vejovsky and Freyer (1994). Because of this, the question arises: Can general theory of non-Abelian gauge fields be used for description of the Abelian version of these fields? Both Verjovsky and Freyer (1994 ) and Trautman (1977) provided affirmative answer to this question. Since the nonperturbative Y-M theory is topological, this then implies that its Abelian version is also topological and, therefore, should be capable of describing some knots/links. Trautman did not discuss knots or links in his paper. Instead, he noticed that the Abelian (that is of U(1)-type)) Y-M connection describing the Dirac monopole is solution of the Maxwell equations very much like the SU(2)-type connection is the solution of the non-Abelian Y-M. He also concluded that the analogous treatment should be applicable to the gauge theory of gravity so that the same computational protocol should yield gravitational instantons. In the light of what follows, this is compatible with the statement that all gauge field theories (Maxwell, Y-M and gravity) should contain solutions describing knots/links. In this paper we shall not discuss gravity-related topics. Interested reader should be able to find relevant information in (Kholodenko 2011).

\subsection{Connection with works by Ranada}

By restricting ourself to the Maxwell and Y-M cases our treatment of instantons in this work (which will be called part I) and its companion (which will be called part II) formally 
differs (but equivalent) from that developed by Trautman. The difference is caused by our desire to discuss from the instanton perspective results of influential paper by Ranada (1989) written much later. It had been in use in the majority of theoretical and experimental works aimed at describing and detecting knotted beams of light. The latest reference (Keida et al 2013) summarizes the latest efforts in this direction. Ranada's original paper as well as many other subsequently written either by him or with collaborators develop formalism without any reference to (connection with) the non-Abelian theory of Y-M fields. As is well known, due to their nonlinearity, the Y-M fields are much harder to study than the Maxwellian

fields. Numerous attempts to treat pure Y-M fields by the saddle point methods resulted in negative answer to the question about the existence of localized solutions (lumps or solitons) in Minkowski space-time. In (Kholodenko 2011) a list of representative works of various degree of rigor is provided in which this negative answer was obtained. Absence of localized solutions makes it impossible to obtain stable "knotty solutions" of the Y-M equations in Minkowski space-time. The same conclusion holds for the Abelian (Maxwellian) gauge fields as it was recently demonstrated in (Chubukalo et al 2010).

At the same time, in the Euclidean space the Y-M fields do have nontrivial saddle pointtype solutions known as instantons and monopoles. Ranada's knots/links were designed to exist in the Minkowski space-time. Contrary to the recent claims made in physics literature they cannot be immediately compared with the electromagnetic instantons obtained by Trautman (Trautman 1977). In this work we demonstrate that, when properly interpreted, Ranada's knots/links do have a chance to exist.

\subsection{Organization of the rest of this paper}

As it was already stated, our work is made of two parts. The first part is meant to reinterpret known (in physics literature) results for knots and links in the Abelian gauge fields in terms of formalism of contact geometry/topology. In doing so some new results are obtained to be listed below. The second part is more technical and requires some in depth knowledge of contact geometry/topology. It is being hoped that our readers will consult whenever they are in doubt physics oriented monograph by Kholodenko (2013) and, purely mathematically oriented monograph by Geiges (2008).

Since, to our knowledge, Floer's work remains outside the scope of the mainstream physics literature we provide in section 2 the self-contained introduction to Floer's ideas. Section 3 contains some ramifications of general results presented in section 2 aimed at reobtaining with help of Floer's methodology designed for Y-M fields the Arnol'd inequality extensively used in the book by Arnol'd and Khesin (1998) on topological methods in hydrodynamics. Detailed study of this inequality is given in section 4 in the context of hydrodynamics and electromagnetism. In this section we begin to exploit the content of this inequality from the perspective of contact geometry and topology. This allows us to recover the major results of Ranada $(1989,1992)$ and to reinterpret them nontraditionally. Obtained results are aimed at preparing our readers for extension of Ranada's results as well as those by (Keida et al 2013 and Dennis et all 2010) to be discussed in part II. Part I concludes with section 5 providing a summary and the list of tasks to be developed and completed in the future publications. 


\section{Basics of Floer-style treatment of Yang-Mills instantons.}

\subsection{Some basic facts about instantons}

We begin our exposition of Floer's ideas by introducing some notations. Following (Dubrovin et al 1984, Frankel 1997) it is sufficient to begin with the Abelian (electromagnetic) case where the vector fields $\mathbf{E}$ and $\mathbf{B}$ represent various components of the second rank skew-symmetric (electromagnetic) tensor $F_{i k}$. It is determined at each point of, say, Minkowski space-time (of signature $1,-1,-1,-1$ ) as

$$
E_{\alpha}=F_{0 \alpha}, \alpha=1,2,3 .-B^{1}=F_{23}, B^{2}=F_{13},-B^{3}=F_{12}
$$

In terms of these notations, the 2 -form $\mathbf{F}$ is defined in a usual way via

$$
\mathbf{F}=\frac{1}{2} F_{i j} d x^{i} \wedge d x^{j}=\sum_{\alpha} E_{\alpha} d x^{0} \wedge d x^{\alpha}-B^{1} d x^{2} \wedge d x^{3}+B^{2} d x^{1} \wedge d x^{3}-B^{3} d x^{1} \wedge d x^{2}
$$

and its dual $* \mathbf{F}$ via

$$
* \mathbf{F}=-\sum_{\alpha} B_{\alpha} d x^{0} \wedge d x^{\alpha}-E_{1} d x^{2} \wedge d x^{3}+E_{2} d x^{1} \wedge d x^{3}-E_{3} d x^{1} \wedge d x^{2}
$$

From here, it follows that $(* \mathbf{F})_{i j}=\frac{1}{2} \varepsilon_{i j l m} F^{l m}, F^{l m}=g^{l p} g^{m q} F_{p q}$ with Minkowski metric tensor $g_{i j}, \varepsilon^{0123}=1$, and $g^{i j}=\left(g_{i j}\right)^{-1}$. With help of these definitions we obtain: $F^{0 \alpha}=-F_{0 \alpha}$, and $F^{\alpha \beta}=F_{\alpha \beta}, \alpha, \beta=1,2,3$. Thus, $*(* \mathbf{F})=-\mathbf{F}$. That is the square of the Hodge star operator in Minkowski space-time is equal to -1 .

The antisymmetric tensor $F_{p q}$ has 6 independent components. These components can be treated as components of some vector in $\mathbf{R}^{6}$. Because of this, introduce complex variable via $\tilde{z}=a+i b$ then, let $\tilde{z} \mathbf{F} \equiv a \mathbf{F}+b * \mathbf{F}$. Under such an identification $i^{2} \mathbf{F}=-\mathbf{F}$. This fact allows us to replace $\mathbf{R}^{6}$ by $\mathbf{C}^{3}$ and to identify the Hodge star operation with that of complex multiplication. In $\mathbf{C}^{3}$ it is possible to introduce complex coordinates $z^{\alpha}$ via

$$
z^{\alpha}=E_{\alpha}+i B^{\alpha}, \alpha=1,2,3 .
$$

Such complex vector is known in literature as Riemann-Silberstein (RS) vector. The Maxwellian theory of electromagnetism was extensively discussed in terms of the RS vector in recent review paper (Bialynicki-Birula 2013). By design, uses of this vector are limited to spacetimes of Minkowski signature. In such a case, one typically introduces the quadratic form

(Dubrovin 1984) $(\mathbf{E}+i \mathbf{B})^{2}=\sum_{\alpha=1}^{3}\left(z^{\alpha}\right)^{2}$ enabling us to classify various possibilities for vector fields $\mathbf{E}$ and $\mathbf{H}$. It is believed (Kedia 2013), that the electromagnetic knots can be formed only by null fields (read, however, Appendix D). These are determined by the condition

$$
\sum_{\alpha=1}^{3}\left(z^{\alpha}\right)^{2}=0
$$


This condition is formally satisfied if $\mathbf{E}+i \mathbf{B}=0$ which is mathematical statement of the antiself-duality in spacetimes of Minkowski signature (Mason and Woodhouse 1996, Chubukalo et al 2010). Clearly, for this to make sense we have to treat both $\mathbf{E}$ and $\mathbf{B}$ as complex numbers thus contradicting the initial assumption made in eq.(2.2). Nevertheless, for the sake of results presented below, it is helpful to know that historically, the concept of (anti)self-duality is associated with the Y-M instantons "living" in Euclidean space. This concept can be used in space-times of Minkowski signature also (Mason and Woodhouse 1996, Nash and Sen 1983, Chubukalo et al 2010). As it was noticed in the Introduction, in such spacetimes the Y-M action functional calculated on (anti)-self-dual fields vanishes. Evidently, this result cannot be immediately applied to the RS vector. Instead, we have to look for another option, e.g.

$$
\mathbf{E}^{2}-\mathbf{H}^{2}+2 i \mathbf{E} \cdot \mathbf{H}=0
$$

where the symbol denotes the Euclidean space scalar product. From here we obtain two conditions: $|\mathbf{E}|=|\mathbf{H}|$ and $\mathbf{E} \cdot \mathbf{H}=0$. These two properties, by design, characterize the null fields. Since any $\mathbf{F}$ can be decomposed as $\mathbf{F}=\mathbf{F}^{+}+\mathbf{F}^{-}$where $\mathbf{F}^{+}=\frac{1}{2}(\mathbf{F}+* \mathbf{F})$ and $\mathbf{F}^{-}=$ $\frac{1}{2}(\mathbf{F}-* \mathbf{F})$, it is clear, that the above null fields can be represented via linear combination of dual and anti-self-dual fields. This option is suggested in (Chubukalo 2010) without any reference to Ranada's results. It happens, however, that Ranada's results indeed fall into exactly this category as will be explained later in the text.

The action functional $S$ for both the Y-M and Maxwellian fields can be written (up to a constant factor) as (Frankel 1996)

$$
S[\mathbf{F}]=-\operatorname{tr} \int_{M}(\mathbf{F} \wedge * \mathbf{F}) \equiv\|\mathbf{F}\|^{2}
$$

Following Floer (Donaldson 2002) it is convenient to design the 4-manifold $\mathcal{M}$ as direct product of some 3 -manifold $Y$ (in the simplest case it is $S^{3}$ ) and time $\mathbf{R}$, that is $\mathcal{M}=Y \times \mathbf{R}$. The sign "-" in front of the integral in eq.(2.5) is written in accord with the Abelian case (Landau and Lifshitz 1975) treated in spaces of Minkowski signature.

From the discussion related to the RS vector it follows that in spacetimes of Minkowski signature the (anti)- self-duality equation/condition is given by

$$
* \mathbf{F}= \pm i \mathbf{F} \text {. }
$$

This result makes sense for both Abelian and non-Abelian gauge fields (Nash and Sen 1983). Switching to spaces with Euclidean signature results in replacing eq.(2.6) by

$$
* \mathbf{F}= \pm \mathbf{F}
$$

In the absence of sources the first pair of Maxwell's equations in Minkowski space-time are given by

$$
\operatorname{div} \mathbf{B}=0, \operatorname{curl} \mathbf{E}+\frac{\partial \mathbf{B}}{\partial t}=0 .
$$


These are equivalent to the condition: $d \mathbf{F}=0$, that is to the Bianchi identity. The second pair of Maxwell's equations are given by

$$
\operatorname{div} \mathbf{E}=0, \operatorname{curl} \mathbf{B}-\frac{\partial \mathbf{E}}{\partial t}=0 .
$$

These are equivalent to the equation $d * \mathbf{F}=0$. Both $d \mathbf{F}=0$ and $d * \mathbf{F}=0$ are obtainable from the action functional, eq. (2.5). These equations look formally the same for both the Y-M and Maxwellian fields. The anti-self-duality equation/condition in the Minkowski space-time is given by

$$
\mathbf{B}=-i \mathbf{E}
$$

while in the Euclidean space it is given by

$$
\mathbf{B}=-\mathbf{E} .
$$

\subsection{Connections with the paper by Ranada (1989). Emergence of dyons}

Formally, the last result, eq.(2.9b), is just eq.(13) of Ranada's paper (Ranada 1989). In fact, Ranada uses both the duality, that is $\mathbf{B}=\mathbf{E}$, and the anti-self duality conditions. Such an interpretation of his results is superficial though. It is superficial because eq.(2.9a) is actually written by Ranada as $\mathbf{B}(\theta)=-\mathbf{E}(\phi)$ and, accordingly, $\mathbf{B}(\phi)=\mathbf{E}(\theta)$. Strictly speaking, these are not (anti)-self-dual fields since for such fields the arguments in the above equations should be the same. How then one should understand these equations? Well, using both of the above equations leads to the conditions: $\mathbf{B}(\theta) \cdot \mathbf{E}(\theta)=-\mathbf{B}(\phi) \cdot \mathbf{E}(\phi)$ and $\mathbf{B}^{2}(\theta)=\mathbf{E}^{2}(\phi), \mathbf{B}^{2}(\phi)=\mathbf{E}^{2}(\theta)$. Should the arguments in all these equations be the same, then these equations would indeed describe the null fields. But they are not the same! Again, how then one should understand these equations?

To inject some physics into these thus far formal results, it is helpful to notice that Maxwell's eq.s(2.8) will remain invariant under formal replacement: $\mathbf{E} \rightarrow-\mathbf{B}, \mathbf{B} \rightarrow \mathbf{E}$. These are the electric-magnetic duality transformations discovered by Heviside in 1893 as described in the paper by Mignaco (Mignaco 2001). Maxwell's equations with charges and currents loose this type of invariance, unless the magnetic monopoles are present. Recall, that according to Trautman the U(1) fiber bundle connection corresponding to the magnetic pole is nontrivial. This connection solves Maxwell's equations while the $\mathrm{SU}(2)$ connection solves the non-Abelian Y-M equations yielding the instanton and monopole solutions. In his papers (Ranada 1989, 1992) Ranada indeed obtains the connection and curvature known for the Dirac monopole. But, in addition, by assuming the electric-magnetic duality he treats the electric charges as electric-type monopoles too. As result, if Trautman's electromagnetic field is the field originating from the Dirac monopole, Ranada's electromagnetic field is the field originating from the Abelian dyon, that is from the hypothetical particle which is carrying the magnetic and electric charges simultaneously. Since neither Ranada and his collaborators 
nor those who used his results had recognized Ranada's construction of electromagnetic field as that attributed to the Abelian dyons (Pakman 2000, Negi and Dehnen 2011), in appendix A we provide some very basic information on dyons. The dyonic interpretation of Ranada's equations $\mathbf{B}(\theta)=-\mathbf{E}(\phi)$ and $\mathbf{B}(\phi)=\mathbf{E}(\theta)$ converts them into (anti)-self-duality conditions in Euclidean space. At the same time, since the scalar product in eq.(2.4) is defined in Euclidean space (having in mind $3+1$ decomposition of Minkowski space-time) Ranada's equations, in fact, define the null fields in Minkowski space-time in the sense we just had described. The $3+1$ decomposition used by Floer allows us to understand this apparent peculiarity without difficulty. This is explained in appendix B. At this point the attentive reader would object to our formal dyonic interpretation of Ranada's results since Ranada's papers describe only

the electromagnetic fields without charges. This objection can be removed, however, as follows. For the sake of argument, consider the case of monopoles first. In our book (Kholodenko 2013) we explained in great detail that the (Dirac) monopole can be recreated with help of superconducting ring in which the superconducting current flows. The magnetic field outside the ring does not have sources and thus forms a complementary ring which is linked with the magnetic field located on the surface of the superconducting ring. Thus, we are dealing with the Hopf-type interlocked rings. As result, the magnetic field coming from such ring system is indistinguishable from the field originated from the Dirac monopole. This requires some proof which is given in (Kholodenko 2013), Chapter 3, section 3.5. In Ranada's case the Dirac monopole is being modelled by the system of interlocked magnetic rings. From here, there is no need for the magnetic charge! Because of the electric-magnetic duality, following Ranada, we have to add to these two magnetic rings another two interlocked electric fields. Such interlocked electric rings serve to replace the electric charge. Thus, both electric and magnetic charges can be described in terms of the corresponding interlocked Hopfian rings. Since the field (electric or magnetic) originating from such Hopfian rings is indistinguisheable from that coming from "true" electric or magnetic charges, the presence of such topological formations in electromagnetic field spares us from the necessity to have actual charges. In view of such an interpretation, the situation in the Abelian case now parallels that in the non-Abelian case (Manton and Suttcliffe 2007) where monopoles and dyons are created directly from the sourceless non-Abelian Y-M fields. In the Abelian case in order to obtain a dyon without actually having electric or magnetic charge it is sufficient to construct a system made of two interlocked magnetic rings to which two interlocked electrical rings should be added. Such a system is being described in terms of the electromagnetic field designed by Ranada in 1989. This interpretation of Ranada's results leads to far reaching consequences. They are going to be briefly discussed in section 5 .

\subsection{Back to instantons}

To develop needed formalism we have to finish with general topics related to instantons. Some auxiliary information needed for connecting Floer's arguments with those known in 
physics literature is collected in Appendix A. From this appendix it follows that in Euclidean space the action functional, eq.(2.5), can be written as

$$
S=\frac{1}{2} \int_{\mathcal{M}_{E}} d v(4)\left[\mathbf{E}^{2}+\mathbf{B}^{2}\right]
$$

where $\mathcal{M}_{E}$ stands for 4-manifold of Euclidean signature. Here $d v(4)$ stands for the volume element for such manifold. In the space-time of Minkowski signature the same action $S$ is known (Landau and Lifshitz 1975) as

$$
S=\frac{1}{2} \int_{\mathcal{M}} d v(3,1)\left[\mathbf{E}^{2}-\mathbf{B}^{2}\right]
$$

It is helpful to add few details to these results. First, we notice that the action is determined with accuracy up to some space/time derivative of some scalar function of $\mathbf{E}$ and $\mathbf{B}$ vector fields. The fact that this derivative can be dropped is the result of imposed space-time boundary conditions which should be specified both in Minkowski and Euclidean spaces. Second, it is helpful to recall that if $\mathcal{M}=Y \times \mathbf{R}$ in eq.(2.10b), then (up to a constant)

$$
\mathcal{E}=\frac{1}{2} \int_{Y} d v(3)\left[\mathbf{E}^{2}+\mathbf{B}^{2}\right]
$$

is the genuine energy density of electromagnetic (Landau and Lifshitz 1975) or Yang-Mills Frankel 1997) fields. By comparing eq.(2.10a) with eq.(2.11) it is evident that in both cases the energy density is the same. This observation will be frequently used in both parts of our work.

To proceed with instantons, in view of eq.(2.5) we also need to introduce the 2 nd Chern number (or instanton topological charge) $C_{2}$ which (up to a constant factor) is given by

$$
C_{2} \simeq \operatorname{tr} \int_{\mathcal{M}}(\mathbf{F} \wedge \mathbf{F})
$$

The constant factor is determined by the gauge group which is in use. Since $\operatorname{tr} \int_{\mathcal{M}}(* \mathbf{F} \wedge \mathbf{F})=$ $\left.\operatorname{tr} \int_{\mathcal{M}}(\mathbf{F} \wedge * \mathbf{F})\right)$, we also obtain,

$$
\operatorname{tr} \int_{\mathcal{M}}(* \mathbf{F} \wedge * \mathbf{F})=\operatorname{tr} \int_{\mathcal{M}}(\mathbf{F} \wedge \mathbf{F}) .
$$

Using this result we rewrite the action in eq.(2.5) as

$$
S[\mathbf{F}]=-\frac{1}{2} \operatorname{tr} \int_{\mathcal{M}}(\mathbf{F}+* \mathbf{F}) \wedge(\mathbf{F}+* \mathbf{F})+\operatorname{tr} \int_{\mathcal{M}}(\mathbf{F} \wedge \mathbf{F}) \geq \operatorname{tr} \int_{\mathcal{M}}(\mathbf{F} \wedge \mathbf{F})
$$

with the equality achieved for the anti-self-dual solutions: $* \mathbf{F}=-\mathbf{F}$. Since it is possible to write the analogous inequality for the dual fields too (Manton and Sutcliffe 2007), use of this inequality is not making the anti-self-dual fields more special than the dual ones. Nevertheless, 
in mathematics literature, e.g. read (Donaldson and Kronheimer 1990), pages 38-39, 43-47, there is an explaination of the fact that in instanton calculations it is mathematically incorrect to use solutions of both duality, that is $\mathbf{F}^{-}=0$, and anti-self-duality, that is $\mathbf{F}^{+}=0$, equations simultaneously. This restriction is associated with the choice of complex structure on the underlying manifold. Once the complex structure is selected, it is mathematically incorrect to switch to another structure. Since the null fields require both duality and anti-self-duality solutions for their realization, this means that Trautman's monopole results (Trautman 1977) are in accord with the existing mathematical restrictions while Ranada's dyonic solution (Ranada 1989,1992) still requires justification which will be provided below.

Next, following Frankel (1997) we write (symbolically) for curvature $\mathbf{F}=d \mathbf{A}+\mathbf{A} \wedge \mathbf{A}$. Therefore, for any curvature 2-form matrix which is defined for any vector bundle over any manifold of any dimension the following chain of equalities holds:

$$
\begin{aligned}
\mathbf{F} \wedge \mathbf{F}= & (d \mathbf{A}+\mathbf{A} \wedge \mathbf{A}) \wedge(d \mathbf{A}+\mathbf{A} \wedge \mathbf{A})= \\
& d \mathbf{A} \wedge d \mathbf{A}+d \mathbf{A} \wedge \mathbf{A} \wedge \mathbf{A}+\mathbf{A} \wedge \mathbf{A} \wedge d \mathbf{A}+\mathbf{A} \wedge \mathbf{A} \wedge \mathbf{A} \wedge \mathbf{A} .
\end{aligned}
$$

By applying the trace operation to the above expression and by keeping in mind that $\operatorname{tr}(\mathbf{A} \wedge$ $\mathbf{A} \wedge \mathbf{A} \wedge \mathbf{A})=0$ and $\operatorname{tr}(d \mathbf{A} \wedge \mathbf{A} \wedge \mathbf{A})=\operatorname{tr}(\mathbf{A} \wedge \mathbf{A} \wedge d \mathbf{A})$, etc. we eventually arrive at the crucial identity

$$
\operatorname{tr}(\mathbf{F} \wedge \mathbf{F})=d \operatorname{tr}\left(\mathbf{A} \wedge d \mathbf{A}+\frac{2}{3} \mathbf{A} \wedge \mathbf{A} \wedge \mathbf{A}\right) .
$$

This identity, when being used in eq.(2.14), leads to the following result:

$$
\begin{aligned}
C_{2} & \simeq \operatorname{tr} \int_{\mathcal{M}}(\mathbf{F} \wedge \mathbf{F})=\int_{\mathcal{M}} \operatorname{trd}\left(\mathbf{A} \wedge d \mathbf{A}+\frac{2}{3} \mathbf{A} \wedge \mathbf{A} \wedge \mathbf{A}\right) \\
& =\int_{\partial \mathcal{M}} \operatorname{tr}\left(\mathbf{A} \wedge d \mathbf{A}+\frac{2}{3} \mathbf{A} \wedge \mathbf{A} \wedge \mathbf{A}\right) \risingdotseq C S(\mathbf{A}) .
\end{aligned}
$$

Here $C S(\mathbf{A})$ stands for the "Chern-Simons functional" and $\risingdotseq$ means "up to a constant" (determined by the gauge group being in use). This derivation assumes that $\mathcal{M}$ is the manifold with boundary. It is not limited to 3 dimensions. The CS-like functionals exist for manifolds of any odd dimensions and, accordingly, the Y-M-like fields can be defined on even dimensional manifolds of dimensionality one higher.

The results just obtained imply

$$
S[\mathbf{F}] \geq \varkappa C S(\mathbf{A})
$$

where $\varkappa$ is some gauge group-dependent constant factor. Therefore, it follows that the minima of $S[\mathbf{F}]$ are determined by the minima of $C S(\mathbf{A})$. These are given by the zero curvature condition (valid at the boundary $\partial \mathcal{M}$ )

$$
\frac{\delta C S(\mathbf{A})}{\delta \mathbf{A}}=\mathbf{F}[\mathbf{A}]=d \mathbf{A}+\mathbf{A} \wedge \mathbf{A}=0
$$

obtainable by minimization of $C S(\mathbf{A})$. Details of minimization calculations can be found, for example, in (Jost 2008). The obtained result leads to the apparent contradiction. Indeed, 
the $C S(\mathbf{A})$ functional is not invariant with respect to gauge transformations (Donaldson 2002, Manton and Sutcliffe 2007). This causes $C S(\mathbf{A})$ to be determined only with accuracy up to some integer. In addition, the field $\mathbf{A}$ used in $S[\mathbf{F}]$ depends upon four independent (space-time) variables while the field $\mathbf{A}$ in $C S(\mathbf{A})$ apparently depends upon 3 variables only. How these difficulties are being resolved in physics literature we shall not discuss in what follows. Instead, we are going to use Floer's ideas, To facilitate understanding of Floer's work 3 , we recommend reading of appendix A inspired by Floer's ideas prior to reading the rest of this section.

\subsection{Basics of Floer's input to the $Y$-M instantons}

Our exposition of Floer's ideas is based in part on results from the Donaldson's monograph (Donaldson 2002). From this work it follows that it is very helpful to discuss the minimization problem for Y-M fields in the context of classical mechanics. For this purpose, it is necessary to reformulate known in physics mechanical formalism somewhat.

Thus, let $q_{i}(t)$ be the i-th component of coordinate describing the trajectory of a particle of unit mass in the potential $V$. Then, Newton's equations of motion are given by

$$
\frac{d^{2}}{d t^{2}} q_{i}=-\nabla_{i} V
$$

These equations are obtainable by variation of the action functional $S[q(t)]$ defined by

$$
S[q(t)]=\int_{0}^{t} d \tau\left[\frac{1}{2} \dot{q}^{2}-V(q)\right] .
$$

The unexpected twist in this well known protocol originates from the following observation. Suppose that there is some function $\sigma(q)$ such that the potential $V$ can be represented as

$$
V=-\frac{1}{2}|\nabla \sigma|^{2}
$$

Then, the first order equation

$$
\dot{q}_{i}=\nabla_{i} \sigma
$$

will have the same solutions as Newton's eq.(2.19).

To prove that this is indeed the case, let us consider the following chain of equalities:

$$
\frac{d}{d t} \dot{q}_{i}=\frac{d}{d t}\left(\nabla_{i} \sigma\right)=\sum_{j} \frac{\partial}{\partial q_{j}}\left(\frac{\partial \sigma}{\partial q_{i}}\right) \frac{d q_{j}}{d t}
$$

By combining this result with eq.(2.22) we obtain:

$$
\frac{d}{d t} \dot{q}_{i}=\sum_{j} \frac{\partial^{2} \sigma}{\partial q_{i} \partial q_{j}} \frac{\partial \sigma}{\partial q_{j}} .
$$

\footnotetext{
${ }^{3}$ That is that part of Floer's works which is needed for the purposes of this paper.
} 
At the same time,

$$
\frac{\partial}{\partial q_{i}} V=-\frac{1}{2} \frac{\partial}{\partial q_{i}} \sum_{j}\left(\frac{\partial \sigma}{\partial q_{j}}\right)^{2}=-\sum_{j} \frac{\partial^{2} \sigma}{\partial q_{i} \partial q_{j}} \frac{\partial \sigma}{\partial q_{j}} .
$$

Using this result in (2.19) we reobtain (2.24) as required. This fact allows us to rewrite the action functional $S[q(t)]$ in the form analogous to eq.(A.5) for the Y-M fields. Indeed, we obtain,

$$
\begin{aligned}
S[q(t)] & =\int_{0}^{t} d \tau\left[\frac{1}{2} \dot{q}^{2}-V(q)\right]=\int_{0}^{t} d \tau \frac{1}{2}\left[\dot{q}^{2}+|\nabla \sigma|^{2}\right] \\
& =\int_{0}^{t} d \tau \sum_{i}\left[\frac{1}{2}\left\{\left[\dot{q}_{i}^{2}-\nabla_{i} \sigma\right]^{2}\right\}+\frac{\partial \sigma}{\partial q_{i}} \frac{d q_{i}}{d \tau}\right] \\
& =\int_{0}^{t} d \tau\left[\frac{1}{2}\left\{|\dot{q}-\nabla \sigma|^{2}+\frac{d \sigma}{d \tau}\right] .\right.
\end{aligned}
$$

Thus, when $\dot{q}_{i}=\nabla_{i} \sigma$ we obtain,

$$
S[q(t)]=\sigma(\tilde{q}(t))-\sigma(q(0))
$$

where $\tilde{q}(t)$ is the solution of eq(2.22) at time $t$. Therefore,

$$
S[q(t)] \geq[\sigma(\tilde{q}(t))-\sigma(q(0))] .
$$

Now by rewriting eq.(A.5) as

$$
S[\mathbf{A}]=\frac{1}{2} \int_{-\infty}^{\infty} d t \int_{Y} d v\left[\dot{\mathbf{A}}^{2}+\mathbf{B}^{2}\right]
$$

since $\boldsymbol{\nabla} \times \mathbf{A}=\mathbf{B}$ and by comparing it against eq.(2.26a) we obtain the following correspondences

$$
q_{i} \rightleftarrows A_{i}, \frac{\partial}{\partial q_{i}} \rightleftarrows \frac{\partial}{\partial A_{i}},|\nabla \sigma|^{2}=\nabla \sigma \cdot \nabla \sigma \rightleftarrows \mathbf{B} \cdot \mathbf{B}
$$

Furthermore, using eq.(2.18) we can write as well

$$
\nabla \sigma \cdot \nabla \sigma \rightleftarrows \sum_{i} \frac{\delta C S(\mathbf{A})}{\delta A_{i}} \frac{\delta C S(\mathbf{A})}{\delta A_{i}}
$$

so that

$$
\sigma \rightleftarrows C S(\mathbf{A})
$$

This requires us to prove that

$$
\frac{\delta C S(\mathbf{A})}{\delta A_{i}}= \pm B_{i}
$$

This task is accomplished in appendix B. But, if eq.(2.31) is correct then, using eq.s $(2.22)$ and (2.31) we obtain as well

$$
\frac{\partial A_{i}}{\partial t}= \pm B_{i}
$$


To decide which sign to choose we have to look at the interpretation of this result in the Abelian case. For such a case we should use the anti-self-duality, eq(2.9), and, from appendix A, the fact that $\mathbf{E}=-\frac{\partial}{\partial t} \mathbf{A}$. This then helps us to reach the conclusion that we must choose the sign " +" in the above anti-self-duality equation. Naturally, it is equivalent to eq.(2.9) as required. In view of eq.(2.31) it is convenient to rewrite eq.(2.32) in the form of the gradient flow equation

$$
\frac{\partial A_{i}}{\partial t}=\frac{\delta C S(\mathbf{A})}{\delta A_{i}}
$$

Since eq.s (2.22) and (2.33) are equivalent, we can interpret both of them as describing critical dynamics of some kind of statistical system. More on this can be found in (Kholodenko 2008) 4 . Eq.(2.33) is nontrivial and leads to the extremely sophisticated analysis done by Floer (1988). Fortunately, we do not need his analysis in this work. Instead, it is helpful now to reconsider once again eq.(2.28). We obtain,

$$
\begin{aligned}
S[\mathbf{A}] & =\frac{1}{2} \int_{-\infty}^{\infty} d t \int_{Y} d v(3)\left[\dot{\mathbf{A}}^{2}+\mathbf{B}^{2}\right]=\frac{1}{2} \int_{-\infty}^{\infty} d t \int_{Y} d v(3)\left[\mathbf{E}^{2}+\mathbf{B}^{2}\right] \\
& =\int_{-\infty}^{\infty} d t \int_{Y} d v(3)\left\{\frac{1}{2}|\mathbf{E}+\mathbf{B}|^{2}-\mathbf{E} \cdot \mathbf{B}\right\} .
\end{aligned}
$$

Deser and Teitelboim (1976) noticed that the $\mathbf{E} \cdot \mathbf{B}$ term in the above integral can be represented as the total divergence, i.e. $\partial_{\mu} C^{\mu}$ where $C^{\mu}=\varepsilon^{\mu \nu \rho \sigma} A_{\nu} \partial_{\rho} A_{\sigma}$. Because of this, when the anti-self-duality condition, eq(2.9.b), is fulfilled, we obtain:

$$
S[\mathbf{A}]=\int_{-\infty}^{\infty} d t \int_{Y} d v \mathbf{B}^{2} \geq C S\left(\mathbf{A}_{\infty}\right)-C S\left(\mathbf{A}_{-\infty}\right)
$$

It makes physical sense to require $\mathbf{A}_{-\infty}=0$ so that $C S\left(\mathbf{A}_{-\infty}\right)=0$. This then leads us to the recovery of the already known result, eq.(2.17). As plausible as it is, this fact is not sufficient for the obtained results to be used further. Explanations are provided in the next section.

\section{Ramifications}

To move forward, we must take into account that the CS functional is not an invariant of gauge transformations. To deal with this fact we need to introduce several definitions. First, we would like to remind to our readers that the A-field (the connection) under gauge transformations is transformed as

$$
\mathbf{A} \rightarrow \mathbf{g A g}-\mathbf{d g g}^{-1} \equiv \mathbf{g}(\mathbf{A})
$$

where $\mathbf{g} \in \mathcal{G}$ where $\mathcal{G}$ is the gauge group of automorphisms of whose Lie algebra is determined by the known set of generators $T_{\alpha}$. As it follows from the above definition, $\mathbf{g}$ is coordinatedependent in general. Because of this, we have to operate not with the space $\mathcal{A}$ of all

\footnotetext{
${ }^{4}$ Interested readers are encouraged also to consult (Oh 2013) and (Sreets 2007).
} 
connections but with its quotient $\mathcal{B}=\mathcal{A} / \mathcal{G}$ (roughly equivalent to the moduli space). $\mathcal{B}$ is a smooth infinite-dimensional manifold $\sqrt{5}$. The moduli space is the subset $\mathcal{R} \subset \mathcal{B}$ defined as the set of all flat connections via

$$
\mathcal{R}=\{[A] \in \mathcal{B} \mid \mathbf{F}[\mathbf{A}]=0\} .
$$

It is invariant with respect to the action of $\mathcal{G}$. We can temporarily fix the constant in the CS functional, eq.(2.15), if, following Floer (1988), we define the CS functional as

$$
\frac{1}{2} \int_{\partial M} \operatorname{tr}\left(\mathbf{A} \wedge d \mathbf{A}+\frac{2}{3} \mathbf{A} \wedge \mathbf{A} \wedge \mathbf{A}\right)=C S(\mathbf{A}) .
$$

Then, gauge transformations defined by eq.(3.1) when applied to $C S(\mathbf{A})$ produces

$$
C S(\mathbf{g}(\mathbf{A}))=C S(\mathbf{A})+2 \pi \operatorname{deg}(\mathbf{g}),
$$

where $\operatorname{deg}(\mathbf{g})$ is some integer. It is the degree of a map between 3-dimensional closed manifolds. Based on this result, Floer concludes that $C S(\mathbf{A})$ is well defined on the quotient

$$
\tilde{\mathcal{B}}=\mathcal{A} /\{\mathbf{g} \in \mathcal{G} \mid \operatorname{deg}(\mathbf{g})=0\}
$$

The gradient flow, eq.(2.33), is well defined only on $\tilde{\mathcal{B}}$. Imposition of the constraint, eq.(3.5), is the major source of differences in treatments of Y-M theory in physics and mathematics. In this work we follow mathematician's path as stated already.

The condition, eq.(3.5), is easy to understand. Indeed, the minimization of eq.(2.18) produces some flat connection $\mathbf{F}[\mathbf{A}]=0$. It belongs to the space $\mathcal{R}$. This connection must be such that the action, eq.(2.3), stays finite. For this reason and the fact that dynamics takes place in Euclidean space, such connections are called instantons. In fact, these are identical with the gauge equivalence classes, say, $a$ and $b$, defined as follows. Recall that instantons in the usual quantum mechanics travel from one hump/lump/maximum (critical point $\equiv$ vac uum) of the inverted potential to another (critical point, that is another vacuum). The maxima are critical points of the underlying manifold. This fact is used in the Morse theory to recover some topological characteristics of the underlying manifold. In the Y-M theory the maxima are determined by eq.(2.18) and the CS functional is playing a role of the Morse function. In such interpretation $a$ and $b$ are different (in general) equivalence classes associated with different critical points of $\mathcal{R}$. Furthermore, it is possible to decompose $\mathcal{R}$ into subspaces $\mathcal{M}(a, b)$ of instantons connecting $a$ and $b \in \mathcal{R}$. Thus, Floer extended the conventional Morse theory so that it becomes capable of computing the topological properties of $\mathcal{R}$. Evidently, if the restriction given by Eq.(3.5) is not imposed, then one cannot talk about well defined classes $a$ and $b$.

Eq.(3.4) is the statement of periodicity of the CS functional. Following Donaldson (2002) this periodicity can be equivalently stated as follows. For the sake of argument we shall use the gauge group $S U(2)$. Then,

$$
C_{2}=\frac{1}{8 \pi^{2}} \operatorname{tr} \int_{[0, t] \times Y}(\mathbf{F} \wedge \mathbf{F}) \equiv \theta(t) .
$$

\footnotetext{
${ }^{5}$ We skip the fact that $\mathcal{B}$ is Banach manifold whose definition is given in (Donaldson 2002)
} 
Accordingly,

$$
\theta\left(t_{2}\right)-\theta\left(t_{1}\right)=\frac{1}{8 \pi^{2}} \operatorname{tr} \int_{\left[t_{1}, t_{2}\right] \times Y}(\mathbf{F} \wedge \mathbf{F}) \bmod \mathbf{Z},
$$

where $\mathbf{Z}$ denotes the ring of integers. In view of this equation and, taking into account eq.(2.15), we obtain (for any gauge group!)

$$
\operatorname{tr} \int_{[0,1] \times Y}(\mathbf{F} \wedge \mathbf{F})=\int_{Y} \operatorname{tr}\left(\mathbf{A} \wedge d \mathbf{A}+\frac{2}{3} \mathbf{A} \wedge \mathbf{A} \wedge \mathbf{A}\right)
$$

This result should be understood as follows. Choose a connection $\mathbf{A}$ on the principal $G$ bundle over 4-manifold $[0,1] \times Y=S^{1} \times Y$ in such a way that for $\{0\} \times Y$ it is trivial while for $\{1\} \times Y$ it is not trivial. Then we have to consider the degree of the map $S^{1} \rightarrow S^{1}$, where $S^{1}=[0,1]$ is mapped into const $\operatorname{CS}(\mathbf{A}(1))$. Here const depends upon the gauge group being used so that the combination const $\mathrm{CS}(\mathbf{A}(1))$ is an integer multiplied by $2 \pi$ (e.g. see eq.(3.4)).

By looking at eq.(3.7) we notice that on the l.h.s. the integration is done over the volume and time while on the r.h.s. only over 3 -volume. To resolve this difficulty, Donaldson suggests to use the following parametrization

$$
\mathbf{A}(t, \mathbf{y})=t A(\mathbf{y})
$$

It should be noted that:

a) since $t \in[0,1]$, we can parametrize $t$ as $t=f(x),-\infty<x<\infty$ so that $\mathbf{A}(-\infty) \in a$ and $\mathbf{A}(+\infty) \in b$. This is 3 dimensional interpretation of $3+1$ dimensional instanton construction.

b) in 4 dimensional interpretation, we have to keep in mind that in temporal gauge $t$ plays a role of parameter. The well known (in physics literature) 1-instanton solution (Polyakov 1987, Donaldson 2002)

$$
|F|=\frac{1}{\left(1+r^{2}\right)^{2}}
$$

obtained on $\mathbf{R}^{4}$, when viewed in the $3+1$ setting in which $\mathbf{R}^{4}$ is replaced by $S^{3} \times \mathbf{R}$, acquires different (but equivalent) look

$$
|F|=\frac{4}{\cosh ^{2}(t)} .
$$

Now $r=r(f(t)), r^{2}(t)=x(t)^{2}+y(t)^{2}+z(t)^{2}+t^{2}$. At $t=-\infty$ a path $\mathbf{r}(t)$ begins at trivial connection for which we have $|F|=0$. Then, for $t \rightarrow \infty$ the path winds once (and only once!) in the space of connections $\mathcal{B}$ and returns again to the flat connection.

Eq.(3.8) allows us to replace the inequality (2.35) by

$$
\int_{Y} d v \mathbf{B}^{2} \geq \mathfrak{N C S}(\hat{\mathbf{A}}) .
$$


where $\mathfrak{N}$ is some constant. Since the connection $\mathbf{A} \equiv \hat{\mathbf{A}}$ must satisfy zero curvature eq.(2.18), we can exploit this fact in order to write

$$
d \hat{\mathbf{A}}=-\hat{\mathbf{A}} \wedge \hat{\mathbf{A}} .
$$

Using this result in the CS functional we obtain (up to a constant)

$$
C S(\hat{\mathbf{A}})=-\frac{1}{3} \int_{Y} \operatorname{tr}(\hat{\mathbf{A}} \wedge \hat{\mathbf{A}} \wedge \hat{\mathbf{A}}) .
$$

Such a form of the CS functional is used in physics literature (Manton and Sutcliffe 2007). There is yet another way to write the same functional. Indeed, using eq.(3.12) in eq.(2.16) we obtain as well

$$
C S(\hat{\mathbf{A}})=\frac{1}{3} \operatorname{tr} \int_{Y} \hat{\mathbf{A}} \wedge d \hat{\mathbf{A}} .
$$

By combining eq.s(3.11) and (3.14) we obtain the result of central importance for our work:

$$
\int_{Y} d v \mathbf{B}^{2} \geq \mathbb{N} \int_{Y} \operatorname{tr}(\hat{\mathbf{A}} \wedge d \hat{\mathbf{A}}),
$$

where $\mathbb{N}$ is yet another positive constant. The discussion of this inequality starts in the next section.

\section{Arnol'd inequality, Ranada's electromagnetic tensor and contact geometry of monopoles and dyons}

\subsection{Arnol'd inequality}

Using results of appendix $\mathrm{C}$, the Abelian version of inequality (3.15) can be rewritten in hydrodynamics language (Kholodenko 2013) where it is known as Arnol'd inequality (Arnol'd and Khesin 1998)

$$
\int_{Y} d v \mathbf{v}^{2} \geq \mathbb{N} \int_{Y} d v \mathbf{v} \cdot(\nabla \times \mathbf{v})
$$

This inequality can be equivalently written as

$$
\mathcal{E}[\mathbf{v}]=\int_{Y} d v \mathbf{v}^{2} \geq \mathbb{C} \int_{Y} d v\left(\mathbf{v} \cdot \operatorname{curl}^{-1} \mathbf{v}\right) \equiv \mathbb{C H}[\mathbf{v}] .
$$

The constant $\mathbb{C}$ is determined in Arnol'd and Khesin's book. It will be also discussed further below. Thus, the helicity $\mathcal{H}[\mathbf{v}]$ provides the lover bound for the (kinetic) energy functional $\mathcal{E}[\mathbf{v}]$. Independently, one can pose the problem: Find the minimum of $\mathcal{E}[\mathbf{v}]$ and the extremals among the velocity field obtained from a given divergence-free field $\mathbf{v}$ by the action of volumepreserving diffeomorphisms. Arnol'd proved the following

Theorem 4.1. The extremals of the just stated problem are divergence-free vector fields that commute with their vorticities. In particular, they coincide with steady Euler flow in $Y$. 
The content of this theorem should be understood as follows. Define the commutator (the analog of Poisson brackets) for the vector fields $\varphi$ and $\psi$ as $\{\boldsymbol{\varphi}, \boldsymbol{\psi}\}=(\boldsymbol{\varphi} \cdot \boldsymbol{\nabla}) \boldsymbol{\psi}-(\boldsymbol{\psi} \cdot \boldsymbol{\nabla}) \boldsymbol{\varphi}$ then, it can be demonstrated that $\{\boldsymbol{\varphi}, \boldsymbol{\psi}\}=\operatorname{curl}(\boldsymbol{\varphi} \times \boldsymbol{\psi})-\boldsymbol{\varphi}(\operatorname{div} \boldsymbol{\psi})+\boldsymbol{\psi}(\operatorname{div} \boldsymbol{\varphi})$. By applying this identity to the divergence-free fields we obtain,

$$
\{\boldsymbol{\varphi}, \boldsymbol{\psi}\}=\operatorname{curl}(\boldsymbol{\varphi} \times \boldsymbol{\psi})
$$

If the fields $\boldsymbol{\varphi}$ and $\boldsymbol{\psi}$ commute, we get $\boldsymbol{\varphi} \times \boldsymbol{\psi}=\boldsymbol{\nabla} \alpha$. When this is rewritten in hydrodynamic language, this result acquires the form of equation describing stationary Euler flow

$$
\mathbf{v} \times \operatorname{curl} \mathbf{v}=\nabla \alpha
$$

with the Bernoulli function $\alpha=\mathcal{P}+\frac{\mathbf{v}^{2}}{2}$. This result is in accord with eq.(C.3a). When translated into electromagnetic language, the analogous result is given by eq.(C.7) of Appendix C

$$
\mathbf{E}+\mathbf{v} \times \mathbf{B}=-\nabla \Phi
$$

But, since thus far we were working with the source-free Maxwell fields the particle(or fluid) velocity $\mathbf{v}$ in (C.7) creates some problem in establishing fluid mechanics-electrodynamics correspondence. This issue was studied first by Newcomb (1958). The latest attempt at resolving this issue can be found in the paper by van Enk (2013).

Before switching to electrodynamics, it is educational to discuss the obtained results in hydrodynamics setting. This is justified because in this case the solutions to eq.(4.2) were analyzed rigorously by Arnol'd (Arnol'd and Khesin 1998).

The analysis begins with the case $\alpha=$ const in eq.(4.2). This condition leads to the so called force-free family of solutions 6 . These are the solutions of the eigenvalue problem

$$
\operatorname{curl} \mathbf{v}=\kappa \mathbf{v}
$$

where the scalar function $\kappa$ may or may not be a constant. Suppose it is not a constant, then using eq.(4.3) we obtain: $\operatorname{div} \kappa \mathbf{v}=\mathbf{v} \cdot \boldsymbol{\nabla} \kappa=0$. We would like to remind to our readers what this equation actually means. If $\kappa=\kappa(x, y, z)=$ const is an equation describing surface and if $\mathbf{r}(\mathrm{t})$ is some trajectory $\mathbf{r}(\mathrm{t})=\{\mathrm{x}(\mathrm{t}), \mathrm{y}(\mathrm{t}), \mathrm{z}(\mathrm{t})\}$ on this surface, then $\frac{d}{d t} \kappa(x(t), y(t), z(t))=$ $v_{x} \kappa_{x}+v_{y} \kappa_{y}+v_{z} \kappa_{z}=\mathbf{v} \cdot \nabla \kappa=0$. That is the condition $\mathbf{v} \cdot \nabla \kappa=0$ means that the fluid velocity $\mathbf{v}$ is always tangential to the surface $\kappa(x, y, z)=$ const, e.g. read (Dubrovin et al 1984). Since the vector field $\mathbf{v}$ is assumed to be nowhere vanishing, the surface $\kappa(x, y, z)=$ const can only be torus $\mathrm{T}^{2}$. The field lines of $\mathbf{v}$ on $\mathrm{T}^{2}$ should be closed if const is rational number. Thus, the force-free condition, eq.(4.3), provides us with the condition for existence of all possible torus knots for rational $\kappa^{\prime} s$ (Gilbert and Porter 1994).

Corollary 4.2. The force-free vector fields defined by eq.(4.3) minimize $\mathcal{E}[\mathbf{v}]$.

\footnotetext{
${ }^{6}$ Note the difference in terminology. The "force-free" termin is used in magnetohydrodynamics while in hydrodynamics the termin "Beltrami" is used instead.
} 
Indeed, using eq.(4.3) in (4.1a) produces

$$
\int_{Y} d v \mathbf{v}^{2} \geq \kappa \mathbb{N} \int_{Y} d v \mathbf{v}^{2}
$$

so that the equality is achieved when $\kappa \mathbb{N}=1$. Details of the proof are given in (Arnol'd and Khesin 1998).

Being armed with hydrodynamical results, now we must look at the analogous interpretation for the elelectrodynamical eq.(C.7). Evidently, the electrodynamics problem is reducible to that we just discussed if we select the potential $\Phi$ in such a way that $\nabla \Phi+\mathbf{E}=0$. This choice happens to be permissible as explained in Appendix D. If this is so, we have to decide what to do with the equation $\mathbf{v} \times \mathbf{B}=\mathbf{0}$. Fortunately, this equation was studied in (Kholodenko 2013) so that here we provide only the summary of results.

First, we notice that $\mathbf{B}=\boldsymbol{\nabla} \times \mathbf{A}, \nabla \cdot \mathbf{A}=0$. Second, we need to assume that $\mathbf{v}= \pm \gamma \mathbf{A}$ where $\gamma$ is some real constant. Using these facts, we obtain:

$$
\mathbf{A} \times \boldsymbol{\nabla} \times \mathbf{A}=\mathbf{0} .
$$

This leads us back to the equation analogous to eq.(4.3), that is we have

$$
\nabla \times \mathbf{A}=\kappa \mathbf{A} .
$$

Clearly, now we can apply all results related to eq.(4.3) to the present case. But, in addition, by applying the curl operator to both sides we obtain as well

$$
\nabla \times \mathbf{B}=\kappa \mathbf{B}
$$

which is the force-free equation. Eq.(4.5a) can be altrernatively rewritten in the form of the London-type equation of superconductivity 7

$$
\nabla \times \mathbf{v}=\kappa \mathbf{v}
$$

and, if this is so, the vortices in superconductors and superfluids fall in the same category as beams of light. Thus, the already discussed connection beween monopoles and superconducting rings comes to play immediately.

\subsection{Helicity and contact geometry}

Obtained results bring us into position when we can discuss Ranada's electromagnetic tensor. The most optimal way to reobtain Ranada's tensor is trough use of the helicity. Since Ranada uses Clebsch variables, we begin with the observation made by Bretheron

\footnotetext{
${ }^{7}$ For the proof that this is indeed the London-type equation, please, read (Kholodenko 2013), page 3.
} 
(1970). Written in electromagnetic language, his argument goes as follows (appendix D). Let $\mathbf{A}=\nabla \varphi+\alpha \nabla \beta$, so that $\mathbf{B}=\nabla \times \mathbf{A}=\boldsymbol{\nabla} \alpha \times \boldsymbol{\nabla} \beta$. Using these facts, the helicity reads as

$$
\begin{aligned}
\mathcal{H}[\mathbf{A}] & =\int_{V} d v \mathbf{A} \cdot \mathbf{B}=\int_{V} d v\{\nabla \varphi \cdot \nabla \alpha \times \nabla \beta\} \\
& =\int_{V} d v \nabla \cdot\{\varphi \boldsymbol{\nabla} \alpha \times \nabla \beta\} \\
& =\int_{\Sigma} d \vec{\Sigma} \cdot(\nabla \alpha \times \nabla \beta) \varphi=0 .
\end{aligned}
$$

The last line comes from identifications: $\mathbf{A} \rightleftarrows \mathbf{v}, \mathbf{B} \rightleftarrows \tilde{\boldsymbol{\omega}}$ and observation that $\tilde{\boldsymbol{\omega}} \cdot \tilde{\boldsymbol{\Sigma}}=\mathbf{0}$ on the surface of the vortex tube (Saffman 1995). Thus, it looks like use of Clebsch variables is restricted to non entangled flows. It can be also demonstrated that Clebsch variables cannot be used when the vorticity (or magnetic field) vanishes in some regions of space (Boozer 2010), (Graham and Heney 2000). The way out of this difficulty was found already in the classical paper by Seliger and Whitham (1968). The same result was rediscovered by Goncharov and Pavlov (1997) and later by Yoshida (2009). Alternative treatment was given by Kuznetsov and Mikhailov (1980).

For the sake of space, we shall discuss only results by Yoshida (2009) since they can be immediately linked with contact geometry. Yoshida notices/proves that for an arbitrary vector field $\mathbf{u}$ it may not be possible to find 3 scalars (scalar functions) $\alpha, \beta, \varphi$ so that representation $\mathbf{u}=\boldsymbol{\nabla} \varphi+\alpha \boldsymbol{\nabla} \beta$ is defined globally in space. That is the map $\left(u_{1}, u_{2}, u_{3}\right) \rightarrow$ $(\varphi, \alpha, \beta)$ may not be injective. The situation can be corrected if instead the generalized form $\mathbf{u}=\nabla \varphi+\sum_{J=1}^{\delta} \alpha_{J} \nabla \beta_{J}$ is being used with $\delta=D-1$ and $D$ being the dimensionality of space. Incidentally, when $D=3$, we obtain

$$
\mathbf{u}=\nabla \varphi+\alpha_{1} \nabla \beta_{1}+\alpha_{2} \nabla \beta_{2}
$$

This result was obtained for the first time by Seliger and Whitham (1968), and rediscovered later by Goncharov and Pavlov (1997). Following Yoshida (2009) and also Marsden and Weinstein (1983) we rewrite eq.(4.7) in terms of differential 1-form

$$
d u=d \varphi+\sum_{j=1}^{m} \alpha_{j} d \beta_{j}
$$

easily recognizable as contact 1-form (Kholodenko 2013, Geiges 2008). For readers with standard physics equation we notice that the familiar from mechanics relation $p_{i}=\frac{\partial S}{\partial q_{i}}$ can be rewritten as the kernel (that is $\omega=0$ ) of the contact 1-form $\omega=d S+\sum_{j=1}^{m} p_{j} d q_{j}$. Because of this, it is clear that $\omega$ should be invariant with respect to canonical transformations as stated in appendix D. But, in addition, in contact geometry there are contactomorphic transformations which can be understood as follows. Let $m=1$ in the above 1-form $\omega$ and 
consider a transformation $(q, S, p) \rightarrow\left(q^{\prime}, S^{\prime}, p^{\prime}\right)$. It is considered as contactomorphic if there is a function $\rho: \mathbf{R}^{3} \rightarrow \mathbf{R}$ which is nowhere zero and such that

$$
d S^{\prime}+p^{\prime} d x^{\prime}=\rho(q, S, p)(d S+p d x) .
$$

The connection with symplectic mechanics is achieved via relation

$$
d \omega=\sum_{j=1}^{m} d p_{j} \wedge d q_{j}
$$

It is clear that the smallest dimension of the symplectic manifold is 2. Accordingly, the smallest dimension of the contact manifold is 3 . A contact structure on 3-manifold $M$ is a smoothly varying plane field $\xi$ (made of planes $\omega=0$ ) which is completely nonintegrable 8 , that is

$$
\omega \wedge d \omega \neq 0 .
$$

Consider now the simplest Clebsch representation written in 1-form language, e.g. $\omega=$ $d \varphi+\alpha d \beta$. Then $d \omega=d \alpha \wedge d \beta$ is the symplectic volume (area) 2 -form. It can be defined both in $\mathbf{R}^{2}$ and on $S^{2}$. Evidently, $S^{2}$ is just one point compactification of $\mathbf{R}^{2}$. Below we shall investigate both cases 9 . For now, we notice that the Bianchi identity $d \mathbf{F}=0$ for Maxwell's eq(2.8a) can be interpreted symplectically. Indeed, if we label the 2-form in eq.(4.10) as $\mathbf{F}$, then, surely, for such defined $\mathbf{F}$ we obtain $d \mathbf{F}=0$. But we already know that $d \omega=d \alpha \wedge d \beta$. Therefore we reobtain back $\boldsymbol{\nabla} \alpha \times \boldsymbol{\nabla} \beta=\mathbf{B}$ as required. Notice that it is this $\mathbf{B}$ which is present in the formula for helicity, eq.(4.6). Now we can rewrite the formula for helicity $\mathcal{H}[\mathbf{A}]$ alternatively as

$$
\mathcal{H}[\mathbf{A}]=\int_{V} \omega \wedge d \omega .
$$

in accord with eq.(3.15). By doing so, we run into contradiction: on one hand, in view of eq.(4.11) $\mathcal{H}[\mathbf{A}]$ must be nonzero, while on another, according to eq.(4.6) it should be zero. The best way out is by relating this problem with that for Dirac's monopole.

\subsection{From Dirac monopoles to Ranada's dyons}

In our discussion of this topic we follow (Kholodenko 2013, Arnol'd and Khesin 1998 and Ryder 1980). We begin with Example 1.19 in (Arnol'd and Khesin 1998). Let F be arbitrary area 2-form on $S^{2}$ normalized by the condition $\int_{S^{2}} \mathbf{F}=1$. Such a form is closed on $S^{2}$ but it is not exact as we shall demonstrate momentarily. Consider a (Hopf) mapping $\pi: S^{3} \rightarrow S^{2}$, then the pullback $\pi^{*} \mathbf{F}$ produces 2 -form which is exact on $S^{3}$. That is if there is 1-form $\omega$ on $S^{3}$ such that $d \omega=\pi^{*} \mathbf{F}$, then

$$
\mathcal{H}[\mathbf{A}]=\int_{V} \omega \wedge \pi^{*} \mathbf{F}
$$

\footnotetext{
${ }^{8}$ For details regarding this concept, please, consult (Kholodenko 2013, Geiges 2008)

${ }^{9}$ Albeit not with equal amount of details
} 
is the Hopf invariant. Since it is a winding (linking) number it is an integer. This statement is formulated as Proposition 1.20. in the book by Arnol'd and Khesin (1998). No proof of this proposition is given in this reference. Only a hint. A proof is given in (Kholodenko 2013). Special cases are discussed below.

The 2-form on $S^{2}$ is given in (Ryder 1980) in the context of Dirac monopole. When normalized in accord with Arnol'd and Khesin, this form is given by $\mathbf{F}=\frac{1}{4 \pi} \sin \theta d \theta \wedge d \phi$. As it is shown in (Eguchi et al 1980) an attempt to represent $\mathbf{F}$ as $\mathbf{F}=d A$ on the entire 2-sphere is useless. Instead it is possible to subdivide $S^{2}$ into the Nothern and Southern parts so that $\mathbf{F}=d A_{ \pm}$for these parts respectively with

$$
A_{ \pm}=\frac{1}{4 \pi r} \frac{1}{z \pm r}(x d y-y d x) .
$$

In spherical coordinates and for the sphere of unit radius we obtain instead

$$
A_{ \pm}=\frac{1}{4 \pi}( \pm 1-\cos \theta) d \phi .
$$

Both expressions are easily recognizable as vector potentials associated with the Dirac monopole. Using these potentials we obtain indeed $\mathbf{F}=d A_{ \pm}$.

The above results were obtained for $S^{2}$. Now we would like to demonstrate that for $\mathbf{R}^{2}$ the results are different. Indeed, take 1-form $\omega=d S+p d x$. From here $\mathbf{F}=d(p d x)=$ $d p \wedge d x$. Accordingly, $\int_{R^{2}} d p \wedge d x=\int_{\partial R^{2}} p d x=0$ since any contour can be squeezed to zero (Cauchy's theorem). Alternatively, this result is coming from the fact that the relationship $p_{i}=\frac{\partial S}{\partial q_{i}} \equiv f\left(x_{1}, \ldots, x_{i}, \ldots\right)$ for $p_{i}$ being assigned describes the Lagrangian manifold. Therefore, for such manifold we must have $\sum_{i} \oint p_{i} d x_{i}=0$ since $d p \wedge d x=\frac{d p}{d x} d x \wedge d x$. Thus, $\mathbf{R}^{2}$ is not good for our purposes and we have to work with $S^{2}$. Next we have to find 1-form $\omega$ on $S^{3}$ such that $d \omega=\pi^{*} \mathbf{F}$ where $\mathbf{F}=\frac{1}{4 \pi} \sin \theta d \theta \wedge d \phi$. The 1-form $\omega$ on $S^{3}$ is contact, as is well known. Therefore, (up to a constant) its standard form is given by

$$
\omega=\sum_{i=1}^{2}\left(x_{i} d y_{i}-y_{i} d x_{i}\right)
$$

Using it, we obtain (again, up to a constant)

$$
d \omega=2\left(d x_{1} \wedge d y_{1}+d x_{2} \wedge d y_{2}\right) .
$$

Next, we introduce the complex variables $z_{0}=x_{1}+i y_{1}$ and $z_{1}=x_{2}+i y_{2}$. In terms of these variables the 2 -form $d \omega$ is written now (up to a constant) as (Bott and Tu 1982)

$$
d \omega=\frac{i}{2 \pi}\left(z_{1} d z_{0}-z_{0} d z_{1}\right) \wedge\left(\bar{z}_{1} d \bar{z}_{0}-\bar{z}_{0} d \bar{z}_{1}\right)
$$

By keeping in mind that the equation for the 3-sphere of unit radius is

$$
\left|z_{0}\right|^{2}+\left|z_{1}\right|^{2}=1
$$


we can rewrite eq. $(4.15 b)$ as

$$
d \omega=\frac{i}{2 \pi} \frac{\left(z_{1} d z_{0}-z_{0} d z_{1}\right) \wedge\left(\bar{z}_{1} d \bar{z}_{0}-\bar{z}_{0} d \bar{z}_{1}\right)}{\left(\left|z_{0}\right|^{2}+\left|z_{1}\right|^{2}\right)^{2}}
$$

In such a form it is valid on $S^{3}$. Now it remains to demonstrate that this result can be presented as $d \omega=\pi^{*} \mathbf{F}$. For this purpose, following (Bott and Tu 1982) it is sufficient to define $z=z_{0} / z_{1} \in S^{2}$ so that in terms of $z$ the above 2 -form can be rewritten as

$$
\mathbf{F}=\frac{i}{2 \pi} \frac{d z \wedge d \bar{z}}{\left(1+|z|^{2}\right)^{2}}
$$

This is the 2-form suggested by Ranada $(1989,1992)$ for the electromagnetic tensor. The connection with electromagnetism follows from the fact that by design this 2-form satisfy the Bianchi identity $d \mathbf{F}=0$ revealing its symplectic origin as explained in subsection 4.2.

To complete this subsection we still have to discuss several issues. We begin with demonstration that the 2 -form $\mathbf{F}=\frac{1}{4 \pi} \sin \theta d \theta \wedge d \phi$ and that given in eq.(4.16) are indeed equivalent. For this purpose, we notice that in spherical coordinates $z=\cos \theta$ so that (up to sign) previous result can be rewritten as $\mathbf{F}=\frac{1}{4 \pi} d \phi \wedge d z$. This is the standard symplectic 2-form on $S^{2}$ discussed in detail in (Kholodenko 2013). By design (e.g. read above) it obeys the normalization condition

$$
\int_{S^{2}} \mathbf{F}=\frac{1}{4 \pi} \int_{0}^{2 \pi} d \phi \int_{0}^{2} d z=1 .
$$

The 2-form in eq.(4.16) is also discussed in detail in (Kholodenko 2013). It is the standard Fubini-Study 2-form on $S^{2}$ In this case the 2-sphere as symplectic manifold is being interpreted as complex projective line $\mathbf{C P}^{1}$ (recall that $z=z_{0} / z_{1}$ ) (Arnol'd 1989). In polar coordinates it can be rewritten as (up to normalization constant)

$$
\mathbf{F}=\frac{1}{2 \pi} \frac{d \phi \wedge r d r}{\left(1+r^{2}\right)^{2}}
$$

It coincides with the 2 -form $\Omega$ presented in the book by Arnol'd (1989) as a Problem 1 on page 347. Evidently the 2-form defined in eq.s(4.16),(4.17) and $\mathbf{F}=\frac{1}{4 \pi} d \phi \wedge d z$ are equivalent. This is so because symplectic mechanics can be rewritten in terms of dynamics in complex projective space $\mathbf{C P}^{n}$ (Arnol'd 1989). The sphere $S^{2}$ is just $\mathbf{C P}^{1}$. These projective spaces are examples of complex Kähler manifolds. From here, every Kähler manifold is also a symplectic manifold. The opposite is not true as it was demonstrated by Thurston (1976). In the present case the 2-form, eq.(4.16), is nesessarily symplectic and, in view of the Darboux theorem, it can be brought to the standard symplectic 2 -form, that is to $\mathbf{F}=\frac{1}{4 \pi} d \phi \wedge d z$. To demonstrate this explicitly, we have to normalize the form in eq.(4.17) correctly. That is we 
have to make sure that $\int_{S^{2}} \mathbf{F}=1$.This leads us to determination of the constant $C$ using the equation

$$
\frac{C}{2 \pi} \int_{0}^{2 \pi} d \phi \int_{0}^{\infty} \frac{r d r}{\left(1+r^{2}\right)^{2}}=1 .
$$

Evidently,

$$
\int_{0}^{\infty} \frac{r d r}{\left(1+r^{2}\right)^{2}}=\frac{1}{2} \int_{1}^{\infty} \frac{d u}{u^{2}}=\frac{1}{2}
$$

Therefore, $C=2$. Using this result, we can now compare these 2-forms. Specifically, we obtain:

$$
\frac{d z}{2}=\frac{d u}{u^{2}} \text { or } \frac{1}{2} z=-\frac{1}{u}+g
$$

with $g$ being yet another constant. Since $u=1+r^{2}$, and for the sphere of unit radius $z$ ranges between 0 and 2, the constant $g$ is easily determined. Specifically, $g=1$. Thus these 2 -forms are indeed equivalent. In view of this fact, the connection of Randada's results with those for Dirac monopoles (Ryder 1980) is established. It will be reobtained at more advanced level in part II. Since Ranada (1992) uses two types of helicities- electric and magnetic- all results for magnetic (Dirac) monopoles can be transferred without change to the electric-type monopoles. If the electric monopole is located at the same place of spacetime as the magnetic monopole, we obtain a dyon. That is a hypotetical particle possessing simultaneously electric and magnetic charges. Thus, in accord with results of appendix A, the electromagnetic field designed by Ranada (1989) is that originating from dyons. As it was explained in section 2.2., both monopoles and dyons are made of interlocked magnetic and electric Hopf-type rings. Thus, in accord with Ranada, there is no need to use the actual charges for description of monopoles or dyons. Furthermore, in part II we shall be dealing with complements of knots/links. According to Thurston, now proved, geometrization conjecture there are 8 geometries for 3-manifolds. As in two dimensional case, one first have to study spaces of positive, zero and negative curvatures and then groups of isometries in these spaces. In 3 dimensions situation changes since complements of knots/links initially embedded in $S^{3}$ create spaces of positive, zero and negative curvatures depending on knot link topology, that is on the fundamental group of the knot/link complement (Scott 1983). Thus, if we believe Einstein, only physical masses can create curvatures. Now, with Dirac monopoles interpreted as interlocked magnetic rings and Ranada's dyons need in masses not only disappear but even becomes problematic.

Next, we need to demonstrate that the 2 -forms given by eq.s(4.7),(4.8) and eq.(4.14) are equivalent. This is so because in the 1st case we have the 1-form $\omega=d \varphi+\alpha_{1} d \beta_{1}+\alpha_{2} d \beta_{2}$ so that $d \omega$ (up to a constant) coincides with that given in eq.(4.15a). This means that using such 1-form we can recover Ranada's $\mathbf{F}$. Just defined contact 1-form lives in $\mathbf{R}^{5}$ while that given by eq.(4.14) lives on $S^{3}$. But $S^{3}$ is embedded in $\mathbf{R}^{4}$ and $\mathbf{R}^{4}$ is symplectic space with symplectic 2 -form given by eq.(4.15a). It is standard result of contact geometry (Geiges 2008, 
Kholodenko 2013) that it is always possible to represent the contact 1-form $\alpha$ living in $\mathbf{R}^{5}$ as

$$
\alpha=d z+\sum_{i=1}^{2}\left(x_{i} d y_{i}-y_{i} d x_{i}\right)
$$

More accurately, it can be demonstrated (Geiges 2008), page 52, that $\alpha$ is contactomorphic to

$$
\tilde{\alpha}=d z+\sum_{i=1}^{2} x_{j} d y_{j}
$$

From here it follows that 1-forms living in $\mathbf{R}^{5}$ and on $S^{3}$ are fully compatible (contactomorphic). This result provides instant proof of results of Seliger and Whitham (1968), Goncharov and Pavlov (1997) and by Yoshida (2009).

\section{Discussion}

We begin by noticing that Ranada's 2-form, eq.(4.16), is identical with the 2-form, eq.(3.9), obtained via instanton method. This observation is in formal accord with results by Trautman (1977) who obtained this result only implicitly by using different arguments. From Section 3 we know that such one-instanton solution should have instanton (winding) number equal to one. This is surely the case for the helicity $\mathcal{H}[\mathbf{A}]$, eq.(4.12a), if it is computed using the 1 and 2-forms described in the previous section. Computations done by Bott and Tu (1982), by Ryder (1980) and, by Kuznetsov and Mikhailov (1980), confirm this. Questions arise: a) Can one construct multiinstanton solutions in the Abelian case if such solutions do exist in the non-Abelian Y-M case (Manton and Sutcliffe 2007)? b) What kinds of knots/links, if any, can be associated with the multiinstanton-type solutions if such solutions exist? c) How method of Floer discussed in Section 2 can accommodate the multiinstanton solutions? d) In the case if the above questions are answered affirmatively, how one then should relate them to the fact (Witten 1989) that the Abelian CS field theory is known to be capable of describing only the Hopf-type links and torus-type knots/links? We shall address some of these problems in the companion publication, part II. In it we shall make a heavy use of the force-free (or Beltrami) eq.(4.3). As results of the appendix D indicate, this equation is both relativistically and gauge invariant which makes its treatment apparently easier 10 . From the discussions presented in this paper it should become clear that this equation is inseparable from the Arnol'd inequality and, therefore from the helicity. The helicity by design is the product of contact geometry/topology. Since the existence of nonzero helicity is indicative of existence of magnetic/electric monopoles, it follows that electric and magnetic monopoles can be described purely geometrically. Accordingly, to move beyond current results in physics literature describing knotted beams of light will require us to go much deeper into formalism of contact geometry/topology. For instance, in appendix D we discussed constructions based on use of Clebch variables $\alpha$ and $\beta$ while in the section 4.2. we introduced eq.(4.7) which requires two copies of $\alpha^{\prime} s$ and $\beta^{\prime} s$. If this requirement is ignored, we would end up with negative result summarized in eq.(4.6). Furthermore, Lund and Regge (1976) demonstrated that while eq.s(D.3a) and (D.3b) are correct from the Galilean perspective,

\footnotetext{
${ }^{10}$ In fact, not a bit easier as references to part II indicate.
} 
they are not reparametrization-invariant. When reparamerization invariance is enforced, we end up with vortices modelled by relativistic strings. These should be knotted/linked. Recall that the connection between strings and vortices, had began with ground breaking work by Nielsen and Olessen (1973). Faddeev and Niemi and many other researchers studied such knotted/linked strings within the framework of abelianized QCD. Details and many up-to date references on the Faddeev-Skyrme model describing such knotted/linked structures can be found in (Kholodenko 2011 and Kholodenko 2013). In view of dyonic interpretation of Ranada's results it is very appropriate to finish this part of our work with the following observations.

Both in Y-M and Einsteinian gravity the problem of treatment of extended bodies is extremely difficult, e.g. read (Kholodenko 2013), page 97 and references therein. Einstein was always unhappy with the right hand side of his equations since this side contained masses. The masses do not fit well into his geometrical formalism. It is well known that both Y-M and gravity have monopole-like solutions. Both these theories can be described topologically in terms of loops, knots/links. Depending upon knot/link topology (that is on the first fundamental group of the knot/link complement in $\mathrm{S}^{3}$ ) these complementary spaces will have positive, zero or negative curvatures. According to Einstein curvatures can only be created by masses. In this paper, part I, and in its companion, part II, we provide enough evidence that masses are not nesessary. Interesting attempt to describe the standard model in terms of knots/links is proposed in the series of papers by Finkelstein, e.g. see (Finkelstein 2005). Alternative treatment of both gravity and the standard model in terms of knots and links is given in (Kholodenko 2011).

Acknowledgement The author would like to thank Dr. Zurab K. Silagadze (Budker Institute, Novosibirsk, Russia) for many discussions regarding the content of this paper and for some technical assistance.

\section{Appendices}

Appendix A. Pre quantization of $Y$ - $M$ fields and electric-magnetic duality

Without account of gauge constraints, the action functional $S$ for both Abelian and nonAbelian Y-M fields in space-times of Minkowski signature is given by (Frankel 1997)

$$
S=\frac{1}{2} \int_{-\infty}^{\infty} d t \int_{Y} d v(3)\left[\mathbf{E}^{2}-\mathbf{B}^{2}\right]
$$

Naturally, it coincides with the well known result for the Maxwell fields (Landau and Lifshitz 1975) For these fields $\mathbf{B}=\boldsymbol{\nabla} \times \mathbf{A}$ and $\mathbf{E}=-\frac{\partial}{\partial t} \mathbf{A}-\boldsymbol{\nabla} \varphi$, as is well known, $A_{0}=\varphi$. For both the Abelian and non-Abelian Y-M fields it is convenient to use the temporal gauge in which $A_{0}=0$. In this gauge the above action can be rewritten in the form

$$
S[\mathbf{A}]=\frac{1}{2} \int_{-\infty}^{\infty} d t \int_{Y} d v(3)\left[\dot{\mathbf{A}}^{2}-(\boldsymbol{\nabla} \times \mathbf{A})^{2}\right]
$$


where $\dot{\mathbf{A}}=\frac{\partial}{\partial t} \mathbf{A}$. From the condition $\frac{\delta S[\mathbf{A}]}{\delta \mathbf{A}}=0$ it follows that: $\frac{\partial}{\partial t} \mathbf{E}=\boldsymbol{\nabla} \times \mathbf{B}$. The definition of $\mathbf{B}$ guarantees the validity of the condition $\boldsymbol{\nabla} \cdot \mathbf{B}=0$. At the same time, from the definition of $\mathbf{E}$ we obtain another Maxwell's equation: $\frac{\partial}{\partial t} \mathbf{B}=-\boldsymbol{\nabla} \times \mathbf{E}$. The question remains, nevertheless. Will these results reproduce the remaining Maxwell's equation $\boldsymbol{\nabla} \cdot \mathbf{E}=0$ ? This equation is essential for correct formulation of the Cauchy problem for these fields 11 . If it is satisfied for $t=0$, it will be also satisfied for $t>0$. Analysis shows, however, that for $t=0$ the existence of this equation is not a consequence of the remaining equations. Accordingly, it should be imposed as an independent condition. This is primary source of some technical difficulties. Without describing them in full, we sketch some key steps. Specifically, let the vector field A be decomposed as $\mathbf{A}=\mathbf{A}_{\|}+\mathbf{A}_{\perp}$. Since $\mathbf{E}=-\frac{\partial}{\partial t} \mathbf{A}$, we obtain as well $\boldsymbol{\nabla} \cdot\left(\mathbf{E}_{\|}+\mathbf{E}_{\perp}\right)=0$. By design,

$$
\nabla \cdot \mathbf{E}_{\perp}=0
$$

At the same time $\boldsymbol{\nabla} \cdot \mathbf{E}_{\|}$remains to be defined by the initial and boundary data. In view of these conventions it is always possible to choose $\mathbf{A}_{\|}=0$ and to use only $\mathbf{A}_{\perp}$ for quantization. Thus, $S[\mathbf{A}]$ defined in eq.(A.2) can be finally rewritten as

$$
S\left[\mathbf{A}_{\perp}\right]=\frac{1}{2} \int_{-\infty}^{\infty} d t \int_{Y} d v(3)\left[\dot{\mathbf{A}}_{\perp}^{2}-\left(\nabla \times \mathbf{A}_{\perp}\right)^{2}\right] .
$$

so that in eq. (2.10b) we have in fact $d v(3,1)=d v(3) d t$. In such a form it is being used as an action functional in the exponent of the path integral for the Y-M fields in both Abelian and non-Abelian settings (Huang 1982), page 152, (Deser and Teitelboim 1976, Donaldson 2002), We shall not write the subscript $\perp$ from now on (unless specified otherwise) following the existing literature conventions. As in the main text, we need to switch now from the space-time of Minkowski signature to that of Euclidean signature. As it is typically done in quantum mechanics and quantum field theories, this is achieved by replacing $t$ by $-i \tau$ resulting in replacement of the factor $i S$ in the exponent of the path integral by $-S$. The Euclideanized action acquires the following form

$$
S[\mathbf{A}]=\frac{1}{2} \int_{-\infty}^{\infty} d t \int_{Y} d v\left[\dot{\mathbf{A}}^{2}+(\boldsymbol{\nabla} \times \mathbf{A})^{2}\right]
$$

in which $\frac{1}{2} \int_{Y} d v\left[\dot{\mathbf{A}}^{2}+(\boldsymbol{\nabla} \times \mathbf{A})^{2}\right]=\frac{1}{2} \int_{Y} d v\left[\mathbf{E}^{2}+\mathbf{B}^{2}\right]$ is easily recognizable as eq.(2.11) of the main text.

Use of eq.(A.3) allows us to discuss the electric-magnetic duality. Deser and Teitelboim (1976) noticed that although Maxwell's equations without sources are invariant with respect to the duality transformation: $\mathbf{E} \rightarrow \mathbf{B}, \mathbf{B} \rightarrow-\mathbf{E}$, the action functional (A.1) is not. Naively, this fact is not causing any problems when Maxwell's equations are recovered from the action functional $S$ variationally. Nevertheless they and subsequent researchers indicated that such non invariance of $S$ is highly undesirable. To fix the problem we follow the paper by Pakman

\footnotetext{
${ }^{11}$ Essentials on the Cauchy problem for Maxwell and gravity fields can be found in our work (Kholodenko 2011), version 1.
} 
(Pakman 2000). Later on Bunster and Henneaux (Bunster and Henneaux 2011) had reached the same conclusions.

For Maxwellian fields the action given in eq.(2.5) can be written as

$$
S[\mathbf{F}]=-\operatorname{tr} \int_{M}(\mathbf{F} \wedge * \mathbf{F})=-\frac{1}{4} \operatorname{tr} \int_{M} d v F^{\mu v} F_{\mu v}
$$

with $F_{\mu v}[\mathbf{A}]=\partial_{\mu} A_{v}-\partial_{v} A_{\mu}$. From it we get the equations of motion

$$
\partial_{\mu} F^{\mu v}[A]=0
$$

and the Bianchi identities

$$
\partial_{\mu} \tilde{F}^{\mu v}[A]=0
$$

with $\tilde{F}^{\mu v}[A]$ being dual of $F_{\mu v}[\mathbf{A}]$. That is using eq.(2.13) we can rewrite the action $S$ as well as

$$
S[\mathbf{F}]=-\frac{1}{4} \operatorname{tr} \int_{M} d v \tilde{F}^{\mu v} \tilde{F}_{\mu v} .
$$

Next, we introduce new variables $Z$ such that $F_{\mu v}[Z]=\partial_{\mu} Z_{v}-\partial_{v} Z_{\mu}$. Evidently, it is possible the to interpret $\partial_{\mu} \tilde{F}^{\mu v}[Z]=0$ as equations of motion while $\partial_{\mu} F^{\mu v}[Z]=0$ as Bianchi identities. Using these results we can rewrite the action $S$ in the form

$$
S=-\frac{1}{8}\{S[A]+S[Z]\}
$$

But the Lagrangian of such defined action is easily recognizable as Ranada's eq(5) of (Ranada 1989)! Furthermore, according to (Costa-Quintana and Lopez -Aguillar 2012), in this action $\tilde{F}^{\mu v}[Z]=\frac{1}{2} \varepsilon^{\mu \nu \rho \sigma} F_{\rho \nu}[A]$, again, in accord with Ranada (1989). Given this observation, we are not yet done. Following Pakman we represent the action $S$ in still another form given by

$$
S\left[A_{\mu}, F_{\mu v}\right]=\int_{M} d v\left[\frac{1}{4} F^{\mu v} F_{\mu v}-\frac{1}{2} F_{\mu v}\left(\partial_{\mu} A_{v}-\partial_{v} A_{\mu}\right)\right]
$$

where $A_{\mu}$ and $F_{\mu v}$ are being treated as independent variables. In view of eq.(A.3) it is convenient to introduce $\mathbf{E}_{\perp}=\nabla \times \mathbf{Z}$. It is also convenient now to rewite eq.(A.4) as

$$
\begin{aligned}
S\left[\mathbf{A}_{\perp}\right] & =\frac{1}{2} \int_{-\infty}^{\infty} d t \int_{Y} d v\left[-\mathbf{E}_{\perp} \cdot \dot{\mathbf{A}}_{\perp}-\left(\nabla \times \mathbf{A}_{\perp}\right)^{2}\right] \\
& =-\frac{1}{2} \int_{-\infty}^{\infty} d t \int_{Y} d v\left[(\nabla \times \mathbf{Z}) \cdot \dot{\mathbf{A}}_{\perp}+\left(\nabla \times \mathbf{A}_{\perp}\right)^{2}\right]
\end{aligned}
$$

But, in view of eq.(A.9) we can rewrite this result also as

$$
S\left[\mathbf{A}_{\perp}, \mathbf{Z}\right]=-\frac{1}{2} \int_{-\infty}^{\infty} d t \int_{Y} d v\left[(\nabla \times \mathbf{Z}) \cdot \dot{\mathbf{A}}_{\perp}+\left(\nabla \times \mathbf{A}_{\perp}\right)^{2}-\left(\nabla \times \mathbf{A}_{\perp}\right) \cdot \dot{\mathbf{Z}}+(\nabla \times \mathbf{Z})^{2}\right]
$$


To check correctness of this result we can perform independent variation with respect to $\mathbf{Z}$ and $\mathbf{A}_{\perp}$. Using integration by parts several times we arrive at

$$
\dot{\mathbf{E}}=\nabla \times \dot{\mathbf{Z}}=\nabla \times \nabla \times \mathbf{A}_{\perp}=\nabla \times \mathbf{B} ; \dot{\mathbf{B}}=\nabla \times \dot{\mathbf{A}}_{\perp}=-\nabla \times \nabla \times \mathbf{Z}=-\nabla \times \mathbf{E} .
$$

These are Maxwell's equations as required. The action functional, eq.(A.12), is manifestly invariant with respect to duality transformations. Finally, using these results and eq.s(12-13) of (Costa-Quintana and Lopez -Aguillar 2012) we conclude that indeed the equations

$$
\nabla \times \dot{\mathbf{Z}}=\nabla \times \nabla \times \mathbf{A}_{\perp} \text { and } \nabla \times \dot{\mathbf{A}}_{\perp}=-\nabla \times \nabla \times \mathbf{Z}
$$

are describing electromagnetic fields originating from a dyon. More on dyons can be found, for example, in (Negi and Dehnen 2011).

\section{Appendix B 3+1 decomposition of $Y$-M fields}

In this appendix we would like to provide details of derivation/justification of eq.(2.32). For this purpose, following Donaldson (2002) we need first to describe the 4-manifolds to be used. These are different from $\mathbf{R}^{4}$ (or $S^{4}$ ) used in physics literature (Polyakov 1987). Accordingly, the fiber bundle in the present case is also different from that used in physics literature.

Let $\mathrm{Y}_{i}$ be a collection of compact Riemannian 3-manifolds 12 . The index $i(i=1, \ldots, n)$ may contain just 1 entry. Let $U_{i}=Y_{i} \times(0, \infty)$. It is to be called "half-tube". To connect such constructed half tube with the design known in physics, it is helpful to notice that when $Y_{i}=S^{3}$ the half tube $S^{3} \times(0, \infty)$ is conformally equivalent to the punctured 4-ball $\mathbf{B}^{4} \backslash\{0\}$, that is to $\mathbf{R}^{4}$. The $S^{3}$ is called the "cross-section". More generally, it is possible to construct 4-manifold $X$ with tubular ends such that each of these ends is having $S^{3}$ as a cross-section. Then, $X$ is conformally equivalent to a punctured manifold $\tilde{X} \backslash\left\{p_{1}, \ldots, p_{n}\right\}$ where $\tilde{X}$ is being compact (previously we had $\mathbf{B}^{4} \backslash\{0\}$ and $\mathbf{B}^{4}$ respectively). As is well known, both the norm, eq.(2.5), and the instanton, eq.(2.7), are conformally invariant (Donaldson and Kronheimer 1990)

The purpose of such constructed 4-manifolds is exactly the same as in $3+1$ decomposition of spacetimes used in general relativity 13 . Specifically, it is possible to pass from connections on the tube $Y \times \mathbf{R}$ to one -parameter family of connections on $Y$. That is the Euclidean time parametrizes connections on $Y$. Thus, locally, a connection $\mathbf{A}$ over tube is given by the connection matrix

$$
\mathbf{A}=A_{0} d t+\sum_{i=1}^{3} A_{i} d y^{i}
$$

In this expression both $A_{0}$ and $A_{i}$ are functions of $t, y_{1}, y_{2}, y_{3}$. In the temporal gauge $A_{0}=0$ the Euclidean time $t$ becomes a parameter for $A_{i}^{\prime} s$. The Hodge operator $*$ initially defined on 4-manifold can now be adopted to 3-manifold $Y$ via the following prescription. Let $\phi$ be

\footnotetext{
${ }^{12}$ That is 3-manifolds with Euclidean-type signature

${ }^{13}$ For a quick introduction to this topic our readers can consult (Kholodenko 2011), version 1.
} 
1-form on $Y$. Construct next a 2-form $d t \wedge \phi$ on $M$ and consider its dual $*(d t \wedge \phi)$, then since $*$ is acting on half-tube, it is possible to write

$$
*(d t \wedge \phi)=*_{3} \phi
$$

where $*_{3}$ is the Hodge operator acting in $Y$ only. Based on this result, all anti-self-dual forms can be brought into form

$$
\Phi=\phi \wedge d t+*_{3} \phi
$$

To check if this is the desired form, we have to demonstrate that $* \Phi=-\Phi$. But $* \Phi=$ $*\left[\phi \wedge d t+*_{3} \phi\right]=*(\phi \wedge d t)+* *(d t \wedge \phi)=-*(d t \wedge \phi)-\phi \wedge d t=-*_{3} \phi-\phi \wedge d t$ QED.

Now we take the 1-form given in eq.(B.1) and apply $d$ operator to it. Thus, we obtain,

$$
\begin{aligned}
\mathbf{F} & =\sum_{i=1}^{3}\left(\frac{\partial}{\partial t} A_{i}\right) d t \wedge d y^{i}+\sum_{i<j}\left(\frac{\partial}{\partial y_{j}} A_{i}-\frac{\partial}{\partial y_{i}} A_{j}\right) d y^{j} \wedge d y^{i} \\
& =\sum_{i=1}^{3} F_{0 i} d t \wedge d y^{i}+\sum_{i<j} F_{i j} d y^{j} \wedge d y^{i} \\
& =\sum_{i=1}^{3} E_{i} d y^{i} \wedge d t-B_{1} d y^{2} \wedge d y^{3}-B_{2} d y^{3} \wedge d y^{1}-B_{3} d y^{1} \wedge d y^{2}
\end{aligned}
$$

in accord with eq.(2.1b). In arriving at this result we took into account that $\mathbf{E}=-\frac{\partial}{\partial t} \mathbf{A}$ as discussed in appendix A. Evidently, $\mathbf{F}=\Phi$. But we just have demonstrated that $* \Phi=-\Phi$. So, it remains to write down this result explicitly. In Euclidean space the easiest way to obtain the desired result is to consider it in the component-wise form. Specifically, we have to consider only the following anti-self-dual conditions

$$
F_{01}=-F_{23}, F_{02}=-F_{31}, F_{03}=-F_{12} .
$$

By combining eq.s(B.4) and (B.5) we obtain

$$
-E_{i}=\frac{\partial}{\partial t} A_{i}=B_{i}, i=1,2,3
$$

in accord with eq.(2.32). Finally, we take into account that: a) $\frac{\delta C S(\mathbf{A})}{\delta \mathbf{A}}=\mathbf{F}$ and b) $B_{i}=*_{3} F_{0 i}$, so that eq.(2.32) can now be rewritten as

$$
\frac{\partial}{\partial t} A_{i}=*_{3} F_{0 i}
$$

This result is in accord with eq.(2.11) of Donaldson's book (Donaldson 2002) where it was given without derivation.

The above results use essentially $3+1$ decomposition of space-time. Use of such a decomposition allows us to introduce the Hodge operator $*_{3}$. By construction, it will act the in the same way both in spaces of Euclidean and Minkowski signature as noticed already by 
Donaldson 14. The anti-self-duality eq.s (B.6) and (B.7) reflect this fact. We would like to illustrate these statements using Maxwell's equations written in 3 dimensional form. These are given by 15

$$
\begin{gathered}
\operatorname{div} \mathbf{B}=0, \nabla \times \mathbf{E}=-\frac{\partial \mathbf{B}}{\partial t}(\text { Bianchi identity } d F=0), \\
\left.\operatorname{div} \mathbf{E}=0, \nabla \times \mathbf{B}=\frac{\partial \mathbf{E}}{\partial t} \text { (Equations of motion } d * F=0\right) .
\end{gathered}
$$

Let now $-\mathbf{E}=\mathbf{B}$ (see eq.(B.6)). Use this result in eq.(2.8b) to obtain

$$
\nabla \times \mathbf{B}+\frac{\partial \mathbf{B}}{\partial t}=0 \text { or, equivalently, } \nabla \times \mathbf{E}+\frac{\partial \mathbf{E}}{\partial t}=0 .
$$

Since eq.(2.8a) can be rewritten as $\nabla \times \mathbf{E}+\frac{\partial \boldsymbol{\nabla} \times \mathbf{A}}{\partial t}=0$, this brings us back to the already known result: $\mathbf{E}=-\frac{\partial}{\partial t} \mathbf{A}$, thus confirming correctness of the anti-self-duality requirement, eq.(B.6). Just obtained results are outcomes of the correctly posed Cauchy problem for Maxwellian fields.

Appendix C Some facts from hydrodynamics and magnetohydrodynamics of ideal fluids

We shall be concerned only with the incompressible ideal fluids. The incompressibility requires us to impose a constraint: $\operatorname{div} \mathbf{v}=0$, where $\mathbf{v}$ is fluid velocity. For simplicity, let the fluid density $\rho=1$ then, Euler's equation acquires the form

$$
\frac{\partial}{\partial t} \mathbf{v}+\mathbf{v} \cdot \nabla \mathbf{v}=-\nabla \mathcal{P}
$$

where $\mathcal{P}$ is the pressure. Since

$$
\frac{1}{2} \nabla \mathbf{v}^{2}=\mathbf{v} \times \tilde{\boldsymbol{\omega}}+\mathbf{v} \cdot \nabla \mathbf{v}
$$

eq.(C.1) can be rewritten as

$$
\frac{\partial}{\partial t} \mathbf{v}=\mathbf{v} \times \tilde{\boldsymbol{\omega}}-\nabla\left(\mathcal{P}+\frac{\mathbf{v}^{2}}{2}\right)
$$

where the vorticity $\tilde{\boldsymbol{\omega}}=\boldsymbol{\nabla} \times \mathbf{v}$. This equation can be interpreted electrodynamically if we notice that $\nabla \cdot \tilde{\boldsymbol{\omega}}=\nabla \cdot(\nabla \times \mathbf{v})=0$. Thus, we may formally identify $\tilde{\boldsymbol{\omega}}$ with the magnetic field $\mathbf{B}$. Accordingly, the combination $-\boldsymbol{\nabla}\left(\mathcal{P}+\frac{\mathbf{v}^{2}}{2}\right)$ can be identified with the electric field $\mathbf{E}$. Then, eq.(C.3a) acquires the form of Newton's equation for the particle of unit mass and charge moving under the influence of Lorentz force, that is

$$
\frac{d}{d t} \mathbf{v}=\mathbf{E}+\mathbf{v} \times \mathbf{B}
$$

\footnotetext{
${ }^{14}$ E.g. read page 35 (bottom) of (Donaldson 2002)

${ }^{15}$ These two equations of the main text are reproduced here for reader's convenience
} 
By applying the curl operator to eq.(C.3a) we obtain

$$
\frac{\partial}{\partial t} \tilde{\boldsymbol{\omega}}=\boldsymbol{\nabla} \times(\mathbf{v} \times \tilde{\boldsymbol{\omega}}) .
$$

Since $\tilde{\boldsymbol{\omega}} \rightleftarrows \mathbf{B}$ we can rewrite eq.(C.3b) in the form used in ideal magnetohydrodynamics (MHD)

$$
\frac{\partial}{\partial t} \mathbf{B}=\nabla \times(\mathbf{v} \times \mathbf{B}) .
$$

Then, using Maxwell's equation $\nabla \times \mathbf{E}=-\frac{\partial \mathbf{B}}{\partial t}$ we can rewrite eq.(C.5) as

$$
\nabla \times(\mathbf{E}+\mathbf{v} \times \mathbf{B})=0 .
$$

From here we obtain

$$
\mathbf{E}+\mathbf{v} \times \mathbf{B}=-\nabla \Phi .
$$

where $\Phi$ is some scalar potential. Multiplication of both sides by $\mathbf{B}$ produces

$$
\mathbf{B} \cdot \nabla \Phi=-\mathbf{E} \cdot \mathbf{B} .
$$

Using this result we obtain as well (Boozer 2010)

$$
\mathbf{v}=\frac{(\mathbf{E}+\nabla \Phi) \times \mathbf{B}}{\mathbf{B}^{2}}
$$

The null fields are obtained when $\boldsymbol{\nabla} \boldsymbol{\Phi}=\mathbf{0}$ (Irvine 2010). Indeed, when this happens, eq.(C.8) produces $\mathbf{E} \cdot \mathbf{B}=0$. It can be proven (Dubrovin et al 1984) that when $\mathbf{E} \cdot \mathbf{B}=0$ it is always possible to find a frame in which $\mathbf{E}^{2}=\mathbf{B}^{2}$.

Appendix D All about and around equation $\mathbf{E}+\boldsymbol{\nabla} \boldsymbol{\Phi}=\mathbf{0}$.

In Appendix A we decomposed vector fields into longitudinal and transverse parts. That is we had $\mathbf{A}=\mathbf{A}_{\|}+\mathbf{A}_{\perp}$ where $\mathbf{A}_{\|}=\nabla \varphi$ while $\mathbf{A}_{\perp}=\nabla \times \tilde{\mathbf{A}}$. It happens that there are other decompositions as well. One of them is due to Clebsch (Lamb 1945). Such a decomposition is especially useful when equations of hydrodynamics need to be rewritten in terms of Hamiltonian equations of classical mechanics. References (Seliger and Whitham 1968) and (Sudarshan and Mukunda 1974) contain excellent treatments of such Hamiltonization protocol. Clebsch variables were also used in Ranada's paper (Ranada 1989) 16 as well as in most of recent papers on optical knots. Here we discuss them in the context of equation $\mathbf{E}+\boldsymbol{\nabla} \boldsymbol{\Phi}=\mathbf{0}$.

The idea of Clebsch lies in the following. Associate with the vector field $\mathbf{A}(\mathbf{r})$ three scalar functions $\varphi(\mathbf{r}), \alpha(\mathbf{r})$ and $\beta(\mathbf{r})$ so that $\mathbf{A}=\nabla \varphi+\alpha \nabla \beta$. Then, $\nabla \times \mathbf{A}=\boldsymbol{\nabla} \alpha \times \boldsymbol{\nabla} \beta$. It is permissible to make these scalar functions dependent on time $t$. This is done with the

\footnotetext{
${ }^{16}$ Albeit for a different reason
} 
purpose of relating the velocity $\mathbf{v}$ in eq.(C.3b)and (C.5) with Clebsch scalars. Suppose that the vortex tube (Section 4) is described in terms of an equation $f(t, x, y, z)=0$, then it is possible to define the normal $\mathbf{N}$ at each point of this tube: $\mathbf{N}=\mathbf{e}_{x} \frac{d f}{d x}+\mathbf{e}_{y} \frac{d f}{d y}+\mathbf{e}_{z} \frac{d f}{d z}$. The vortex tube can then be described in terms of the equation (Lamb 1945, Saffman 1995) $\tilde{\boldsymbol{\omega}} \cdot \mathbf{N}=\mathbf{0}$ or,

$$
\left[\omega_{x} \frac{\partial}{\partial x}+\omega_{y} \frac{\partial}{\partial y}+\omega_{z} \frac{\partial}{\partial z}\right] f=0
$$

which is typically can be re written as

$$
\frac{d x}{\omega_{x}}=\frac{d y}{\omega_{y}}=\frac{d z}{\omega_{z}}=d t \text { or } \text { as } \frac{d \mathbf{r}}{d t}=\tilde{\boldsymbol{\omega}}(\mathbf{r}(t))
$$

But, $\tilde{\boldsymbol{\omega}}=\boldsymbol{\nabla} \times \mathbf{v}$ and also, $\tilde{\boldsymbol{\omega}}(\mathbf{r}(t))=\boldsymbol{\nabla} \alpha(\mathbf{r}(t)) \times \boldsymbol{\nabla} \beta(\mathbf{r}(t))$. Therefore,

$$
\frac{d}{d t} \alpha(\mathbf{r}(t))=\nabla \alpha(\mathbf{r}(t)) \cdot \frac{d \mathbf{r}}{d t}=\nabla \alpha(\mathbf{r}(t)) \cdot \tilde{\boldsymbol{\omega}}(\mathbf{r}(t))=0
$$

and

$$
\frac{d}{d t} \beta(\mathbf{r}(t))=\nabla \beta(\mathbf{r}(t)) \cdot \frac{d \mathbf{r}}{d t}=\nabla \beta(\mathbf{r}(t)) \cdot \tilde{\boldsymbol{\omega}}(\mathbf{r}(t))=0
$$

These results are compatible with those discussed in section 4.1. Indeed, since $\frac{d \mathbf{r}}{d t}=\mathbf{v}$ and, in view of eq.(D.1b), we obtain: $\mathbf{v}=\nabla \times \mathbf{v}$. This is Beltrami-type equation. The obtained results suggest that both Clebsch scalars $\alpha$ and $\beta$ are constants of motion. The vortex orbit $\mathbf{r}(t)$ is contained in the level sets of both $\alpha(\mathbf{r}(t), t)$ and $\beta(\mathbf{r}(t), t)$. This can be alternatively stated as the requirement that $\mathbf{v}$ is determined by both equations

$$
\frac{\partial}{\partial t} \alpha+\mathbf{v} \cdot \nabla \alpha=0
$$

and

$$
\frac{\partial}{\partial t} \beta+\mathbf{v} \cdot \nabla \beta=0
$$

Since in the MHD laguage $\mathbf{B}=\nabla \alpha \times \nabla \beta$, by multiplying eq.(D.3a) by $\nabla \beta$ and eq.(D.3b) by $\nabla \alpha$ and by subtracting the 2 nd equation from the 1st, the following result is obtained (Stern 1970)

$$
\mathbf{v} \times \mathbf{B}=\nabla \beta\left(\frac{\partial}{\partial t} \alpha\right)-\nabla \alpha\left(\frac{\partial}{\partial t} \beta\right) .
$$

Now, we obtain as well

$$
\begin{aligned}
\frac{\partial}{\partial t} \mathbf{B} & =\nabla\left(\frac{\partial}{\partial t} \alpha\right) \times \nabla \beta+\nabla \alpha \times \nabla\left(\frac{\partial}{\partial t} \beta\right) \\
& =\nabla \times\left[\nabla \beta\left(\frac{\partial}{\partial t} \alpha\right)-\nabla \alpha\left(\frac{\partial}{\partial t} \beta\right)\right] \\
& =\nabla \times(\mathbf{v} \times \mathbf{B})
\end{aligned}
$$


in accord with eq.(C.5). The map $\left(A_{x}, A_{y}, A_{z}\right) \rightarrow(\varphi(\mathbf{r}), \alpha(\mathbf{r}), \beta(\mathbf{r}))$ is not bijective (Yoshida 2009), (Sudarshan and Mukunda 1974) though. Indeed, if $\alpha^{\prime}$ and $\beta^{\prime}$ are functions of $\alpha$ and $\beta$ such that the Jacobian $\frac{\partial\left(\alpha^{\prime}, \beta^{\prime}\right)}{\partial(\alpha, \beta)}=1$, then $\alpha^{\prime}$ and $\beta^{\prime}$ can be used instead of $\alpha$ and $\beta$.The requirement $\frac{\partial\left(\alpha^{\prime}, \beta^{\prime}\right)}{\partial(\alpha, \beta)}=1$ is the same as used in canonical transformations of Hamiltonian mechanics and it is, in fact a canonical transformation as further explained in the main text. Furthermore, it is possible also to make time-depenent transformations of $\alpha$ and $\beta$ which leave $\mathbf{B}$ unchanged. These transformations replace more familiar (to physics educated readers) canonical transformations by the less familiar contactomorphic transformations (Kholodenko 2013, Geiges 2008) discussed in the main text. Boozer (2010) noticed that eq.(C.7) is well behaved only if $\mathbf{B} \cdot \nabla \Phi=-\mathbf{E} \cdot \mathbf{B}$. In such a case eq.(C.9) is obtained. Only if $\nabla \Phi=0$ we reobtain back results used in (van Enk 2013) and (Irvine 2010) leading to null fields.

Because Clebsch transformations belong to a special case of Galilei-type transformation as explained by Sudarshan and Mukunda (1974), the choice $\nabla \Phi=0$ corresponds to a choice of a special reference frame. Another choice of frame in which the vortex is not moving leads to $\mathbf{E}+\boldsymbol{\nabla} \boldsymbol{\Phi}=0$ as required for the validity of arguments of section 4.2. Evangelidis (1988) demonstrated that: a) the force-free condition eq.(4.5b) is both gauge and relativistically invariant; b) if electromagnetic field is described by the pre assigned pair $(\mathbf{E}, \mathbf{B})$ in a given reference frame, it is always possible to find another reference frame in which the electric field $\mathbf{E}$ is parallel to the magnetic field $\mathbf{B}$. Such a frame should move with velocity $\mathbf{v}$ (with respect to the frame with pre-assigned pair $(\mathbf{E}, \mathbf{B})$ ) determined by

$$
\mathbf{v}=(\mathbf{E} \times \mathbf{B}) \frac{c}{2} \frac{\left|\mathbf{E}^{2}\right|+\left|\mathbf{B}^{2}\right|}{\sqrt{\left|\mathbf{E}^{2}\right|\left|\mathbf{B}^{2}\right|-(\mathbf{E} \cdot \mathbf{B})^{2}}}\left\{1 \pm \frac{\sqrt{\left(\left|\mathbf{E}^{2}\right|-\left|\mathbf{B}^{2}\right|\right)^{2}+4(\mathbf{E} \cdot \mathbf{B})^{2}}}{\left|\mathbf{E}^{2}\right|+\left|\mathbf{B}^{2}\right|}\right\}
$$

In the case if the preassigned pair $(\mathbf{E}, \mathbf{B})$ is such that $\left|\mathbf{E}^{2}\right|-\left|\mathbf{B}^{2}\right|=0$, and $\mathbf{E} \cdot \mathbf{B}=\mathbf{0}$ we obtain: $\mathbf{v}=c$. Here $c$ is the speed of light. This is the case of null fields discussed in the text. This conclusion is in accord with that presented in the paper by van Enk (2013). The frame in which $\mathbf{E}$ is parallel to $\mathbf{B}$ was suggested by Chu and Ohkawa (1982) who demonstrated that in this frame the force-free eq.(4.5) holds true. Gray (1992) suggested physical conditions under which one can create a situation in which $\mathbf{E}$ is parallel to $\mathbf{B}$. He also suggested (in a way different from that discussed in Section 4) that this condition leads to the localized configurations of the electomagnetic field. Evangelidis (1988) obtained his results independently from Brownstein (1987) who also came to the same conclusions using different arguments.

\section{References}

Arnol'd V 1989 Mathematical Methods of Classical Mechanics 
(Berlin: Springer-Verlag)

Arnol'd V and Khesin B 1998 Topological Methods in Hydrodynamics

(Berlin: Springer-Verlag)

Arnol'd V 1984 Catastrophe Theory (Berlin: Springer-Verlag)

Arnol'd V 1986 First steps in symplectic topology,

Russian Mathematical Surveys 41 1-21

Bialynicki-Birula I and Bialynicki-Birula Z 2013 The role of the Riemann-Silberstein vector in classical and quantum theories of electromagnetism J.Phys. A 46053001

Birman J and Williams R 1983 Knotted periodic orbits in dynamical systems Topology 22 47-82

Boozer A 2010 Mathematics and Maxwell's equations

Plasma Phys.Control Fusion 52124002

Bott R and Tu L 1982 Differential Forms in Algebraic Topology (Berlin: Springer-Verlag)

Bretheron F 1970 A note on Hamilton's principle for perfect fluids J.Fluid Mech.44 19-31

Brownstein K 1987 Transformation properties of the equation $\nabla \times \mathbf{V}=k \mathbf{V}$ Phys.Rev. A 35 4856-4858

Bunster C and Henneaux M 2011 Can (electric-magnetic) duality be gauged? Physical Review D $\mathbf{8 3 0 4 5 0 3 1}$

Costa-Quintana J and Lopez-Aguilar F 2012 Extended Lagrangian formalisms for dyons and some applications to solid systems under external fields Ann.Phys. 327 1948-1961

Chu C and Ohkawa T 1982 Transverse electromagnetic waves with E $\| \mathbf{B}$ Phys.Rev.Lett.48 837-838

Chubukalo A, Espinosa A and Kosyakov B 2010 Self-dual electromagnetic fields Am.J.Phys.78 858-861

Deser S and Teitelboim C 1976 Duality transformations of Abelian and non-Abelian gauge fields Phys.Rev.D 13 1592-1597

Dennis M, King R, Jack B, O'Holleran K and Padgett M 2010 Isolated optical vortex knots Nature Physics 6 118-121

Donaldson S 2002 Floer Homology Groups in Yang-Mills Theory (Cambridge UK: Cambridge University Press)

Donaldson S and Kronheimer P 1990 The Geometry of Four-Manifolds (Oxford UK: Clarendon Press)

Dubrovin B, Fomenko A and Novikov S 1984 Modern Geometry-Methods and Applications. Part I (Berlin: Springer-Verlag)

Eguchi T , Gilkey P and Hanson A 1980 Gravitation, gauge theory and differential geometry Phys.Rep. 66 213-293

Evangelidis E 1988 Electomagnetic fields satisfying the condition $\mathbf{B} \wedge(\nabla \wedge \mathbf{B})=0$ Astrophysics and Space Science 143, 113-121 
Fischer A and Moncrief V 2001 The reduced Einstein equations and conformal volume collaps of 3-manifolds

Class.Quantum Grav. 18 4493-4515

Finkelstein R 2006 Masses and Interactions of q-Fermionic Knots

Int.J.Mod.Phys.A 21 4269-4302

Floer A 1988 An instanton invariant for 3-manifolds Comm.Math.Phys.

118 215-240

Frankel T 1997 The Geometry of Physics (Cambridge UK: Cambridge University Press)

Geiges H 2008 An Introduction to Contact Topology

(Cambridge UK: Cambridge University Press)

Ghrist R, Holmes P and Sullivan M 1997 Knots and links in three dimensional flows

Lecture Notes in Mathematics $\mathbf{1 6 5 4}$

(Berlin: Springer-Verlag)

Ghys E $2007 \quad$ Knots and Dynamics

Proceedings of the International Congress of Mathematicians

Madrid Spain 2006 pp 247-277

(Zurich: European Mathematical Society)

Gilbert N and Porter T 1994 Knots and Surfaces

(Oxford UK: Oxford University Press)

Goncharov V and Pavlov V 1997 Some remarks on the physical foundation of the Hamiltonian description of fluid motion

Eur.J.Mech. B 16 509-512

Graham R and Heney F 2000 Clebsch representation near points where

the vorticity vanishes Phys.Fluids 12 744-746

Gray J 1992 Electromagnetic waves with E parallel to B

J.Phys.A 25 5373-5376

Helmholtz H 1858 Uber Integrale, der hydrodynamischen Gleichungen, welhe der Wirbelbewegungen entsprehen

J.für die Reine und Angewandte Mathematik 55 25-55

Huang K 1982 Quarks,Leptons and Gauge Fields

(Singapore:World Scientific)

Irvine W 2010 Linked and knotted beams of light, conservation of helicity and the flow of null electromagnetic fields

J.Phys.A 43385203

Jost J 2008 Riemannian Geometry and Geometric Analysis

(Berlin: Springer-Verlag)

Kedia H, Bialynicki-Birula I, Peralta-Salas D and Irvine W 2013

Tying knots in light fields Phys.Rev.Lett 111150404

Kholodenko A 2013 Applications of Contact Geometry and Topology in Physics (Singapore:World Scientific)

Kholodenko A 2011 Gravity assisted solution of the mass gap problem for pure Yang-Mills fields International Journal of Geometric Methods 
in Modern Physics 8 1355-1418

Kholodenko A 2008 Towards physically motivated proofs of the Poincare' and geometrization conjectures J.Geom.Phys. 58 259-290

Kleckner D and Irvine W 2013 Creation and dynamics of knotted vortices in fluid Nature Physics 9 253-258

Kuznetsov E and Mikhailov A 1980 On the topological meaning of canonical Clebsch variables Phys.Lett. A 77 37-38

Lamb H 1945 Hydrodynamics (New York: Dower Publications)

Landau L and Lifshitz E 1975 Classical Theory of Fields

(Oxford UK: Reed Educational and Professional Publishing Ltd.)

Lomonaco S 1996 The modern legacies of Thompson's atomic vortex theory in classical electrodynamics in The Intrface of Knots and Physics pp 145-156 (Providence RI, AMS Publishers)

Manton N and Sutcliffe P 2007 Topological Solitons (Cambridge UK: Cambridge University Press)

Marsden J and Weinstein A 1983 Coadjoint orbits, vortices, and Clebsch variables for incompressible fluids Physica D 7 305-323

Mason L and Woodhouse N 1996 Integrability, Self-duality, and Twistor Theory (Oxford UK: Clarendon Press)

Mignaco J 2001 Electromagnetic duality, charges, monopoles, topology Brazilian J.of Physics 31 235-246

Nash C and Sen S 1983 Topology and Geometry for Physicists (New York: Academic Press Inc.)

Negi O and Dehnen H 2011 Gauge formulation for two potential theory of dyons Int.J.Theor.Phys.50 2446-2459

Nego O and Dehnen H 2011 Gauge formulation for two potential theory of dyons Int.J.Theor.Phys. 50 2446-2459

Newcomb W 1958 Motion of magnetic lines of force Ann.Phys.3 347-385

Nielsen H. and Olesen P 1973 Vortex-line models for dual strings Nucl.Phys. B $\mathbf{6 1} 45-61$

Oh S-J 2013 Finite energy global well-posedness of the $(3+1)$ dimensional Yang-Mills equations using a novel Yang-Mills heat flow gauge $\mathrm{PhD}$ Department of Mathematics Princeton University

Packman R 2000 Schwarz-Sen duality made fully local

Phys.Lett.B 474 309-314

Polyakov A 1987 Gauge Fields and Strings

(New York: Harwood Academic Publishers)

Ranada A 1989 A Topological theory of the electromagnetic field Lett.Math.Phys. 18 97-106

Ranada A 1992 Topological electromagnetism J.Phys.A 25 1621-1641 
Ricca R 2001 An Introduction to the Geometry and Topology of Fluid Flows (Boston: Kluver)

Ryder L $1980 \quad$ Dirac monopoles and the Hopf map $S^{3} \rightarrow S^{2}$

J.Phys.A 13 437-447

Saffman P 1995 Vortex Dynamics (Cambridge UK: Cambridge University Press)

Scott P 1983 The geometries of 3-manifolds

Bull. London Math.Soc. 15 401-487

Seliger R and Whitham G 1968 Variational principles in continuum mechanics Proc.Roy.Soc.A 305 1-25

Stern D 1970 Euler Potentials Am.J.Phys. 38 494-501

Streets J 2007 Ricci Yang-Mills flow PhD Department of Mathematics Duke University

Sudarshan E and Mukunda N 1974 Clssical Dynamics: A Moden Perspective (New York: John wiley\&Sons Inc)

Thompson W 1868 On vortex motion Trans.Roy.Soc.Edinburg 25 217-260

Thompson J 1883 A Treatise on the Motion of Vortex Rings (London:Macmillan)

Thurston W 1976 Some simple examples of symplectic manifolds Proc.Am.Math.Soc. 55 467-468

Trautman A 1977 Solutions of the Maxwell and yang-Mills equations associated with Hopf fibrings International J.of Theoretical Physics 16 561-565

van Enk S 2013 The covariant description of electric and magnetic field lines of null fields: applications to Hopf-Ranada solutions J.Phys.A 46175204

Verjovsky A and Freyer R 1994 The Jones-Witten Invariant for flows on a 3-dimensional manifold Comm.Math.Phys. 163 73-88

Witten E 1989 Quantum field theory and Jones polynomial Comm.Math.Phys. 121 351-399

Yoshida Z 2009 Clebsch parametrization: Basic properties and remarks on its applications J.Math.Phys. 50113101 